\title{
28 Research Square \\ CDR1as related miRNA-mRNA networks in differentiating goat skeletal muscle satellite cells
}

\section{Bismark Kyei}

Sichuan Agricultural University https://orcid.org/0000-0003-1474-5743

\section{Li Li}

Sichuan Agricultural University

\section{Liu Yang}

Sichuan Agricultural University

\section{Siyuan Zhan}

Sichuan Agricultural University

Juntao Li

Sichuan Agricultural University

\section{Yuan Chen}

Sichuan Agricultural University

\section{Xujia Zhang}

Sichuan Agricultural University

\section{Shuailong Zheng}

Sichuan Agricultural University

\section{Xiaoli Xu}

Sichuan Agricultural University

\section{Emmanuel Odame}

Sichuan Agricultural University

\section{Jiaxue Cao}

Sichuan Agricultural University

\section{Jiazhong Guo}

Sichuan Agricultural University

\section{Tao Zhong}

Sichuan Agricultural University

\section{Linjie Wang}

Sichuan Agricultural University

Hongping Zhang ( $\nabla$ zhp@sicau.edu.cn )

\section{Research}


Keywords: Dicer1; CDR1as; Differentiation; Skeletal muscle; Myogenesis

Posted Date: April 29th, 2020

DOI: https://doi.org/10.21203/rs.3.rs-25213/v1

License: (c) (1) This work is licensed under a Creative Commons Attribution 4.0 International License. Read Full License 


\section{Abstract}

Background: Myogenesis is a complex process controlled by several coding and non-coding RNAs (ncRNAs) such as circular RNAs (circRNAs) that well-known function as endogenous microRNAs (miRNAs) sponges. Over the past few years, numerous circRNAs have been known and their roles in biological processes have begun to be understood. Cerebellar Degeneration-Related protein 1 antisense (CDR1as), the most spotlighted circRNA as miR-7 sponge that has been blooming circRNAs' research for a decade, and can potentially sponge several miRNAs in disease and muscle physiology. Nevertheless, the linear-RNAs-differed character that the acute interventions for circRNAs do not affect miRNAs levels, and has retarded the transcriptome-wide discovery of miRNAs sponged by. Therefore, the purpose of this study was to provide the transcriptomic effect of CDR1as during muscle differentiation.

Methods: siCDR1 as and siDICER1 were transfected into goat skeletal muscle satellite cells (SMSCs). RNA-seq technology and bioinformatics tools were used to analyze genes that are deregulated by siCDR1as and siDICER1. quantitative PCR was used to verify the expression levels of the differentially expressed mRNAs and miRNAs.

Results: Here, to systematically identify miRNAs targeting CDR1as, we employed the critical enzyme DICER1 that governs the biogenesis of miRNAs. The deficiency of either DICER1 or CDR1as inhibited myogenic differentiation of SMSCs, and knockdown of DICER1 decreased the expression of CDR1as. Moreover, we screened for the targeted messenger RNAs (mRNAs) and miRNAs in SMSCs transfected with siDICER1 or siCDR1as respectively and found out that some well-known muscle-related pathways such as phosphoinositide 3-kinase (PI3K)-AKT signaling pathway, Rap1 signaling pathway, and MAPK signaling pathway were enriched in all groups. Further, regarding the miRNAs identified in SiDICER1 and siCDR1as together with the sequence complementary information, we identified 11 miRNAs including miR-1, miR-206, and miR-27a-5p which are more likely to be novel targets for CDR1as.

Conclusion: In summary, our study provides a perspective on the potential functions and relationship between CDR1as and DICER1 during muscle development.

\section{Background}

Skeletal muscle is an important component that provides structural support and energy storage, and it is correlated with the quality and quantity of meat production. Skeletal muscle has become an essential material for studying myogenesis in mammals [1]. Myogenesis is the process of skeletal muscle regeneration, which starts with the activation of quiescent satellite cells after injury followed by proliferation, differentiation, and fusion of myoblast into myotubes [2]. Muscle regeneration is controlled by several myogenic regulatory factors such as MyoD, Myf5, myogenin, and MRF4 [3-5] as well as myocyte enhancer factor 2 (MEF2) families including myocyte enhancer factor 2C (MEF2C) [6]. MEF2C is a protein-coding gene that plays an important role in myogenesis of skeletal, cardiac and smooth muscle during muscle differentiation [6-8]. Moreover, according to Alder et al. MEF2C expression is directly 
linked to fusion and myotubes formation [9]. Muscle development is controlled posttranscriptionally through numerous RNA-binding proteins (RBPs) and also non-coding RNAs (ncRNAs) [10-12].

microRNAs (miRNAs) are a small class of non-coding RNAs with the size of about 18-24 nucleotides length that bind to mRNAs of coding genes to repress their protein production. miRNAs are known to play a crucial role in regulating several biological processes, such as myoblast proliferation and differentiation through the control of the targeted mRNAs during muscle development [13]. DICER1 is a ribonuclease III crucial for the biogenesis of mature miRNAs [14]. The deletion of DICER1 generally prevents miRNAs processing and activities. The knockout of DICER1 in mice leads to embryonic lethality related to pluripotent stem cells and imperfect blood vessel formation $[15,16]$. In addition, mature miRNAs produced by DICER1 are shown to be related to the development of the endocrine pancreas and the production of insulin [17]. Moreover, DICER is indicated to modulate miRNAs during myogenic differentiation [18].

Except for linear transcripts like miRNA, IncRNAs, and mRNAs, numerous circular RNAs (circRNAs) responsible for skeletal muscle development have been identified through the introduction of high throughput sequencing technology [19-21]. circRNAs are newly discovered non-coding RNA molecules with a closed-loop structure and acting as gene regulators in most organisms [22, 23]. Although circRNAs also work through regulating alternative splicing or transcriptional mechanism and even by coding micropeptide during several biological processes and diseases [22, 24]. Currently, the most attractive and extensively studied function for circRNAs are sponging miRNAs as a competing endogenous RNA (ceRNA), that is, indirectly elevating miRNA-targeted mRNA genes through direct sponge of those miRNAs and consequently relieve the degradation of mRNA caused by the miRNA [22]. Cerebellar DegenerationRelated Protein 1 antisense RNA (CDR1as), is the most well-studied ceRNAs as a miR-7 sponge (also known as a sponge for miR-7, ciRS-7). Aside from miR-7, other miRNAs such as miR-135a, miR-876-5p and miR-1290 are also potential miRNAs targeting CDR1as in cancer-related diseases [25, 26], and muscle-related diseases [27]. However, the linear-RNAs varied character that the acute interferences for the circRNAs do not have an effect on miRNAs levels [28], and has declined the transcriptome-wide discovery of miRNAs being sponged.

To date, even though some works have been done to establish the fact that CDR1as and DICER play critical roles in muscle development separately [29-31]. The whole transcriptomic effect (including mRNAs and miRNAs) of CDR1 as in myogenic differentiation remains to be elucidated. In this study, we knockdown the expression of CDR1as and DICER1 in skeletal muscle satellite cells (SMSCs), and determined the relationship between CDR1as and DICER1. Further, using RNA-seq we systematically identified differentially expressed patterns of miRNAs and mRNAs associated with the downregulation of CDR1as and DICER1. These results will help us to fully understand the role of CDR1as in SMSCs development and make a paradigmatic example in a systematic study of circRNAs.

\section{Methods}




\section{Cell culture}

The SMSCs used here were successfully isolated from Longissimus dorsi (LD) muscles of newborn goats in our laboratory [32]. SMSCs were cultured in high-glucose Dulbecco's modified Eagle's Medium (DMEM) supplemented with 10\% FBS (Gibco, Grand Island, USA) and 2\% Penicillin \& Streptomycin (Invitrogen, Carlsbad, USA) solution (Growth medium, $\mathrm{GM}$ ) in $5 \% \mathrm{CO}_{2}$. Confluent cells were digested with $0.25 \%$ trypsin including $10 \mathrm{mM}$ EDTA, re-suspended in the corresponding medium, and seeded in 6-well plates with a suitable density. To induce myoblast differentiation, SMSCs were changed to differential medium ( $2 \%$ horse serum, $1 \%$ Penicillin \& Streptomycin (Invitrogen, Carlsbad, USA) and DMEM) in nearly 100\% confluence.

\section{Cell Transfection}

For gain and loss function study, SMSCs with 80\%-90\% confluency in 6-well plates were transfected with Lipofectamine 3000 (Invitrogen, Carlsbad, CA, USA) with siCDR1as, siDICER1 or siNC, at a concentration of $50 \mathrm{nM}$, according to manufacturer's instructions. Moreover, pCDNA-3 was also transfected as a negative control for CDR1as. After $5 \mathrm{hr}$ of transfection, the medium containing transfection reagent was replaced by fresh GM (Growth medium). After culturing for an additional $48 \mathrm{hr}$, cells were used for further experiments. The siCDR1 as and siDICER1 sequence are 5'-GTCTACGATATCCAGGGTT-3' and 5'GCAGTTACGATTTAGCTAA-3' respectively.

\section{Rna Extraction}

Total RNAs were extracted from cells with liquid nitrogen by using RNAiso Plus reagent (TaKaRa Bio, Inc., Japan) and purified using a QIAGEN RNeasy Mini Kit (QIAGEN, Chatsworth, CA, USA) according to the manufacturer's instructions. RNA degradation and contamination were monitored on $1.5 \%$ agarose gels. The purity and concentration of the RNAs were measured using NanoPhotometer ${ }^{\circledR}$ spectrophotometer (IMPLEN, Los Angeles, CA, USA) and a Qubit RNA Assay Kit in a Qubit ${ }^{\circledR} 2.0$ Fluorometer (Life Technologies, Carlsbad, CA, USA), respectively. All RNA samples were stored at $-80^{\circ} \mathrm{C}$ until further use.

\section{Mirna Sequence}

After verifying the concentration and purity, the integrity was assessed using an RNA Nano 6000 Assay Kit in a Bioanalyzer 2100 system (Agilent Technologies, Santa Clara, CA, USA). Only samples that had RNA Integrity Number (RIN) scores > 7.5 were used for RNA sequencing. Briefly, small RNAs were reversed transcribed and amplified by PCR. The PCR products were then purified by denaturing polyacrylamide gel electrophoresis (PAGE). A total of $3 \mu \mathrm{g}$ RNA per sample was used as input material for miRNA sampling preparation. miRNA libraries were constructed and sequencing was performed on an Illumina HiSeq 2500 platform (Illumina, San Diego, CA, USA), and 125-bp long paired-end reads were generated. miRDeep2 
software was used to predict the novel miRNAs with trimmed reads. Then the reads were aligned to merged pre-miRNA databases (known pre-miRNA from miRBase v21 plus the newly predicted premiRNAs) using Novoalign software (v2.07.11) with, at most, one mismatch. We used the most abundant isomiR, the mature miRNA annotated in miRBase and all isoforms of miRNA ( $5 p$ or $3 p$ ) to calculate miRNA expression. Fold change and $p$-value were used to calculate the differentially expressed miRNA profiles between two groups. Hierarchical clustering was performed to generate an overview of the characteristics of expression profiles based on values of a significant differentially expressed transcripts.

\section{Mrna Sequencing Data Processing}

Clean reads were obtained by removing reads containing adapters, reads containing over $10 \%$ of poly $(\mathrm{N})$, and low quality reads ( $>50 \%$ of the bases had Phred quality scores $\leq 10)$ from the raw data. All downstream and upstream analyses were based on high-quality clean data. Goat reference genome and gene model annotation files were downloaded from NCBI database (CHIR_1.0, NCBI) [33]. Index of the reference genome was built using Bowtie v2.0.6 [34, 35] and paired-end clean reads were aligned to the reference genome using TopHat v2.0.14 [36]. The mapped reads from each library were assembled with cufflinks v2.2.1 [37]. We used the reference annotation based transcript (RABT) assembly method in cufflinks v2.2.1 to construct and identify mRNA transcripts from the TopHat2 alignment results. Hierarchical clustering was performed to generate an overview of the characteristics of expression profiles based on values of a significant differentially expressed transcripts.

\section{Kegg Pathway Analysis}

Kyoto Encyclopedia of Genes and Genomes (KEGG) analysis of DEGs was performed with KOBAS software [38] using a hypergeometric test. KEGG pathways with $Q$ value $<0.05$ were considered significantly enriched.

\section{Validation Of Rna-seq Data By Qpcr}

Total RNA was extracted from SMSCs transfected with siCDR1as siNC using RNAiso Plus reagent (TaKaRa Bio, Inc., Japan). For qPCR of mRNA, all PCR primers were designed at or just outside exon/exon junctions to avoid the amplification of residual genomic DNA using the Primer-BLAST on the NCBI website, and specificity was determined using BLASTN. $1 \mu \mathrm{g}$ of total RNAs $(1 \mathrm{mg})$ was reversetranscribed into cDNA by using PrimeScriptTM RT reagent Kit with gDNA Eraser (TaKaRa, Otsu, Japan). And using these cDNA as templates, expression levels of genes were quantified by quantitative real time PCR (RT_qPCR) in a Bio-Rad CFX96 system (Bio-Rad, Hercules, USA) with SYBR Premix Ex TaqTM II (TaKaRa, Otsu, Japan), according to the manufacturer's protocols. Three samples were collected for each treatment and each sample at least triplicates. The PCR protocol was as follows: denaturation at $95^{\circ} \mathrm{C}$ for $30 \mathrm{~s}$, followed by 40 cycles of $95^{\circ} \mathrm{C}$ for $20 \mathrm{~s}$ and $60{ }^{\circ} \mathrm{C}$ for $20 \mathrm{~s}$, then $72{ }^{\circ} \mathrm{C}$ for $30 \mathrm{~s}$. The $2^{-\triangle \Delta \mathrm{Ct}}$ 
procedure was used to calculate the relative expression levels of mRNAs, with GAPDH as an internal control [39]. For qPCR of miRNA, reversed transcribed using The First-strand cDNA Synthesize (TaKaRa, Mount view, CA, USA). For real-time PCR, all reactions were performed in triplicate with SYBR Premix Ex TaqTM II (TaKaRa, Otsu, Japan) under the following conditions: $10 \mathrm{~s}$ at $95^{\circ} \mathrm{C}$ for initial denaturation, followed by 39 cycles of $95^{\circ} \mathrm{C}$ for $5 \mathrm{~s}$ and $60{ }^{\circ} \mathrm{C}$ for $20 \mathrm{~s}$, then melting curve of $65^{\circ} \mathrm{C}$ to $95^{\circ} \mathrm{C}$ for $5 \mathrm{~s}$. The expression levels of U6 were used to normalize the expression levels of the gene of interest. Primers for the mRNAs and miRNAs were designed using Primer-BLAST (http://www.ncbi.nlm.nih.gov/tools/primers-blast) (Table S1 a, b, c, and d).

\section{Statistical analysis}

Data are expressed as mean \pm SEM. All statistical analyses will be performed using GraphPad Prism 6.01. Unpaired student's tests were used to performed statistical analysis used for two group comparisons. Statistical significant difference was evaluated $p<0.05$.

\section{Results}

\section{Effect of siCDR1as and siDICER1 on SMSCs differentiation and the relationship between CDR1as and DICER1}

DICER1 is central to miRNA-mediated silencing [40] and modulates miRNAs during myogenic differentiation [18]. Meanwhile, CDR1as plays a critical role in myogenesis by functioning as a molecular sponge for miR-7 [29]. To identify whether CDR1 as and DICER1 are associated in myoblast differentiation, we in vitro cultured SMSCs isolated from Longissimus dorsi (LD) muscles of newborn Jiangzhou Big-Eared goats and knocked down the expression of CDR1 as and DICER1 (Fig. 1a, d) as well as mouse myoblast C2C12 (Suppl. Figure 1, 2) by using their respective interfering RNAs. Similar to previous results, deficiency of CDR1as or DICER1 decreased the expression of MyoD mRNA, the master myogenic gene (Fig. 1b, e), and the formation of myotubes (Fig. 1c, h). Moreover, CDR1 as was shown to be downregulated in SMSCs transfected with siDICER1 (Fig. 1f) and co-transfection of pCDNA3 (negative control for CDR1as) and siDICER1 (Fig. 1g), suggesting that the close relationship between DICER1 and CDR1as could help identify miRNAs and their targeted mRNAs regulated by CDR1as.

Figure 1 The Expression of Cerebellar Degeneration-Related protein 1 antisense (CDR1as) and DICER1 on skeletal muscle satellite cells (SMSCs) differentiation. (a and d) The expression level of CDR1as and DICER1 in SMSCs transfected with siCDR1 as or siDICER1 was validated. (b and e) qPCR analysis of MyoD expression in SMSCs after transfection with siCDR1as or negative control (NC) and siDICER1 or NC. (c and $h$ ) A representative of microscopic images after the knockdown of CDR1 as and DICER1. ( $f$ and g) SMSCs were transfected with NC or siDICER1, pCDNA3 + NC or pCDNA3 + siDICER1 to determine the relationship between CDR1 as and DICER1. The $p$ values were analyzed by Student's test; $p<0.05$. 


\section{Overview of mRNA and miRNA sequencing data associated with DICER1 and CDR1 as}

In order to predict mRNAs and miRNAs associated with DICER1 and CDR1as in SMSCs, we systematically cultured SMSCs and knocked down the expression of CDR1 as and DICER1, with three biological replicates for each treatment. Cells were harvested at $48 \mathrm{~h}$ after transfection and the total RNA were extracted to construct the cDNA libraries individually for mRNA-seq and miRNA-seq using an Illumina HiSeq 2500 platform and 125 bp paired-end reads.

After removing low-quality sequences and adapters, considering the siCDR1as results, an average of $59,821,102$ and 58,552,463 mRNAs were produced from raw and clean reads respectively (Table 1). In addition, an average of 15,350,087 raw reads and 14,614,375 clean reads miRNAs for siCDR1as were generated respectively (Table 2). On the other hand, siDICER1 has an average of 128,604,571 and $120,968,505$ mRNAs were obtained from raw and clean reads respectively (Table 3 ). Also, an average of $14,260,428$ raw reads and $14,028,682$ clean reads miRNAs for siDICER1 were acquired accordingly (Table 4).

To explore the expression relationship of genes between samples, the Pearson's correlation coefficient (PCC) of mRNAs and miRNAs expression levels of siCDR1as-1, 2 and 3, as well as, siDICER1-1, 2, and 3 in SMSCs were calculated and used to generate a correlation chart. As shown, the correlation coefficient of the siCDR1as-1, 2, and 3 as well as, siDICER1; 1, 2, and 3 in SMSCs ranged from 0.96 to 0.99 (average of 0.98), indicating that the samples replicate very well biologically (Fig. 2a, b, c, and d).

Figure 2 siCDR1as and siDICER1 Pearson correlation chart. (a-d) The abscissa and the ordinate were the respective samples, and the abscissa and the ordinate of each patch represented the correlation of siCDR1as and siDICER1 samples. Importantly, two completely related genomes had a value of 1 . The closer to 1 the relative value is, the larger the Pearson correlation coefficient (PCC) for the siCDR1as and siDICER1 samples; conversely, the closer to 0 the relative value was, the smaller the PCC between the siCDR1as and siDICER1 samples.

To verify the RNA-sequencing data, in extension samples of SMSCs with siDICER1 or siCDR1as, five downregulated as well as five upregulated mRNA and miRNAs identified were randomly selected and quantified for their expression levels using qRT-PCR. As shown in (Fig. 3b; Suppl. Table 2a), (Fig. 4b; Suppl. Table 2b), (Fig. 5b; Suppl. Table 2c) and (Fig. 6b; Suppl. Table 2d), all the differential expression tendency were confirmed.

\section{Differentially expressed (DE) mRNAs and their functional enrichment in SMSCs transfected with siDICER1}


The mRNA expression profiles were detected in SMSCs transfected with siDICER1 and siNC, and hierarchical clustering (Fig. 3a) was performed to show differential mRNA expression patterns among samples. Considering the mRNA expression profiling data, a total of 1,113 mRNAs were differentially expressed in SMSCs transfected with siDICER1 $(p<0.05)$, of which 686 mRNAs (568 known transcript) were downregulated, and 427 mRNAs (365 known) were upregulated. Some myogenic genes including myogenin (MyoG), myocyte enhancer factor 2D (MEF2D) [41], bone morphogenetic protein 4 BMP4 [42], E2F transcription factor 2 (E2F2), insulin-like growth factor binding protein 5 (IGFBP5), cysteine-rich 61 (CCN1) and angiopoietin-1 (ANGPT1) were downregulated.

In addition, KEGG pathway analysis was conducted based on the differentially expressed mRNAs ( $Q$ value $<0.05$ ). The results showed that among the top 20 most enriched KEGG pathways of the downregulatory genes include PI3K-AKT signaling pathway, Focal adhesion, and ECM receptor interaction (Fig. 3c), which are all associated with muscle development. Moreover, considering the upregulatory genes (Suppl. Figure 3), none of the 20 enriched KEGG pathways was associated with muscle development. These data show that the DICER1 gene is involved in the formation of SMSCs.

Figure 3 Expression profile of mRNAs in siDICER1 and NC (SMSCs). (a) Microarray analysis for mRNAs was performed with RNA extracted from siDICER1 $(n=3)$ and NC $(n=3)$ SMSCs. Hierarchical cluster analysis of significantly differentially expressed mRNAs: bright green, under-expression; gray, no change; bright red, over-expression. (b) Ten differentially expressed representative mRNAs were validated in SMSCs siDICER1 and NC by qPCR ( $n=10$ per group). GAPDH was used as an internal control. (c) KEGG of the downregulated mRNAs with the top 20 enrichment. Bubble color and size correspond to the $Q$ value and gene number enriched in the pathway. The rich factor indicates the ratio of the number of DEGs mapped to a certain pathway to the total number of genes mapped to this pathway.

\section{De Mirnas In Smscs Transfected With Sidicer1}

A total of 542 miRNAs were detected in SMSCs samples, among which 22 miRNAs consisting of 7 downregulated and 15 upregulated miRNAs $(F C>1.25$ and padj $<0.05)$ were significantly interfered by Dicer1 (si-DICER1) (Fig. 4a). Notably, myomiRNAs including miR-1, miR-206, and miR-133a/b [43], were upregulated by the deficiency of DICER1. However, miR-133 family contributes to myoblast proliferation, but also prevents differentiation by inhibiting Serum Response Factor (SRF) [13]. miR-1290 was not detected while the expression of miR-135a was almost undetectable in SMSCs treated with siDICER1/siCDR1 as or not. Intriguingly, except for those DE miRNAs mentioned above, miR-7 was the most highly expressed one was insignificantly affected by siDICER1 (Table 5).

Furthermore, using TargetScan, RNAhybrid, and miRanda, a total of 7,194 targets mRNAs were predicted as targets of those upregulated miRNA genes whilst 1,686 target mRNAs of the downregulatory miRNAs were screened. All the mRNAs for the upregulated-miRNAs were enriched in the KEGG pathway such as, Focal adhesion, FoxO signaling pathway, MAPK signaling pathway, PI3K-AKT signaling pathway, Rap1 signaling pathway, and mTOR signaling pathway which are related to muscle development (Fig. 4c; 
Suppl. Figure 4). Moreover, this result is consistent with the functional enrichment for DE mRNAs mentioned before.

Figure 4 Differentially expressed miRNAs in siDICER1 and NC (SMSCs). (a) Sequencing analysis for miRNAs was performed from siDICER1 $(n=3)$ and NC $(n=3)$ of SMSCs. Hierarchical cluster analysis of significantly differentially expressed miRNAs: bright red, overexpression; white, no change; bright blue, under-expression. (b) Differential expression of ten representative miRNAs was validated in SiDICER1 and NC of SMSCs by qPCR ( $n=10$ per group). (c) KEGG analysis of the upregulated miRNA-mRNA network. Bubble color and size correspond to the $Q$ value and gene number enriched in the pathway. The rich factor indicates the ratio of the number of DEGs mapped to a certain pathway to the total number of genes mapped to this pathway.

\section{De Mrnas In Smscs Transfected With Sicdr1as}

From mRNA expression profiling data, a total of 789 mRNAs (Fig. 5a) were differentially expressed in SMSCs transfected with siCDR1as $(p<0.05)$, of which 401 mRNAs were downregulated (316 known protein-coding genes, 85 novel transcripts), and 389 mRNAs were upregulated (277 protein-coding genes, 112 novel transcripts). Some myogenic genes including signal transducer and activator of transcription 2 (STAT2) [44], ANGPT1 [45], intercellular adhesion molecule-1 (ICAM1), E2F2 [46], CCN1 [47], fibroblast growth factor receptor 1 (FGFR1) [48], and MEF2C [49] were downregulated according to the sequencing data.

Further, we performed KEGG pathway analysis with KOBAS software based on the differentially expressed mRNAs genes $(Q$ value $<0.05)$. The results showed that among the top 20 most enriched pathways of the downregulated genes in siCDR1as samples, some well-known muscle-related pathways including PI3K-AKT signaling pathway signaling pathway, Focal adhesion, Rap1 signaling pathway, and MAPK signaling pathway (Fig. 5c) were identified. While those genes up-regulated by siCDR1as were mainly enriched in fatty acid biogenesis and metabolism (Suppl. Figure 5). These results indicate that just like DICER1, the downregulated mRNAs caused by the deficit of CDR1 as are closely related to muscle development.

Figure 5 Expression profile of mRNAs in siCDR1 as and NC (SMSCs). (a) Microarray analysis for mRNAs was performed with RNA extracted from siCDR1as $(n=3)$ and NC $(n=3)$ SMSCs. Hierarchical cluster analysis of significantly differentially expressed mRNAs: bright green, under-expression; gray, no change; bright red, over-expression. (b) Ten differentially expressed representative mRNAs were validated in SMSCs siCDR1as and NC by qPCR ( $n=10$ per group). GAPDH was used as an internal control. (c) KEGG of the downregulated mRNAs with the top 20 enrichment. Bubble color and size correspond to the $Q$ value and gene number enriched in the pathway. The rich factor indicates the ratio of the number of DEGs mapped to a certain pathway to the total number of genes mapped to this pathway. 


\section{De Mirnas In Smscs Transfected With Sicdr1as}

From mRNA expression profiling data, a total of 789 mRNAs (Fig. 5a) were differentially expressed in SMSCs transfected with siCDR1as ( $<$ 0.05), of which 401 mRNAs were downregulated (316 known protein-coding genes, 85 novel transcripts), and 389 mRNAs were upregulated (277 protein-coding genes, 112 novel transcripts). Some myogenic genes including signal transducer and activator of transcription 2 (STAT2) [44], ANGPT1 [45], intercellular adhesion molecule-1 (ICAM1), E2F2 [46], CCN1 [47], fibroblast growth factor receptor 1 (FGFR1) [48], and MEF2C [49] were downregulated according to the sequencing data.

Further, we performed KEGG pathway analysis with KOBAS software based on the differentially expressed mRNAs genes $(Q$ value $<0.05)$. The results showed that among the top 20 most enriched pathways of the downregulated genes in siCDR1as samples, some well-known muscle-related pathways including PI3K-AKT signaling pathway signaling pathway, Focal adhesion, Rap1 signaling pathway, and MAPK signaling pathway (Fig. 5c) were identified. While those genes up-regulated by siCDR1as were mainly enriched in fatty acid biogenesis and metabolism (Suppl. Figure 5). These results indicate that just like DICER1, the downregulated mRNAs caused by the deficit of CDR1as are closely related to muscle development.

Fig. 5 Expression profile of mRNAs in siCDR1as and NC (SMSCs). (a) Microarray analysis for mRNAs was performed with RNA extracted from siCDR1as $(n=3)$ and NC $(n=3)$ SMSCs. Hierarchical cluster analysis of significantly differentially expressed mRNAs: bright green, under-expression; gray, no change; bright red, over-expression. (b) Ten differentially expressed representative mRNAs were validated in SMSCs siCDR1as and NC by qPCR ( $n=10$ per group). GAPDH was used as an internal control. (c) KEGG of the downregulated mRNAs with the top 20 enrichment. Bubble color and size correspond to the $Q$ value and gene number enriched in the pathway. The rich factor indicates the ratio of the number of DEGs mapped to a certain pathway to the total number of genes mapped to this pathway.

\section{Anchoring The Novel Core Mirnas Regulated By Cdr1as}

Considering the major function of DICER1 on modulating miRNAs during myogenesis, and its closely positive effect on CDR1as as shown by expression of CDR1 as as well as the enrichment results. First of all, we investigated the critical miRNAs that mediate the function of DICER1, using online tool MSigDB (http://www.broadinstitute.org/gsea/msigdb/index.jsp) and the DE mRNAs dataset caused by siDICER1. We found out 100 miRNAs were potentially targeted by these DE mRNA genes altered by siDICER1 (FDR q-value < 0.004), among which 11 miRNAs including miR-1, miR-199b-5p, miR-206, miR-27a-5p, miR-19b3p, miR-30b-5p, miR-129-5p, miR-128-3p, miR-30e-5p, miR-27b-5p, and miR-424-5p were overlapped with those differentially expressed miRNAs detected by using miRNA-seq in SMSCs transfected with siDICER1. This indicates that these miRNAs may play important roles in mediating DICER's function in myogenic differentiation of SMSCs. 
Furthermore, six miRNAs including miR-1, miR-206, miR-424-5p, miR-30b-5p, miR-128-3p, and miR-19b-3p were enriched in targeting the upregulated mRNA genes caused by interfering CDR1as (siCDR1as) while only miR-199a was enriched in downregulated genes too (Fig. 8a; Suppl. Table 3). Additionally, miR-146a, miR-19a/b, miR-27a-5p, and miR-30e-5p were overlapped in DE miRNAs caused by si-DICER1 and siCDR1as, suggesting that the following 11 miRNAs including miR-1, miR-206, miR-424-5p, miR-30b-5p, miR-128-3p, miR-19b-3p, miR-199a, miR-146a, miR-19a/b, miR-27a-5p, and miR-30e-5p, are much more likely to be novel target for CDR1as. Further, RNAhybrid was used to predict the complementary interactions between CDR1 as and these miRNAs. The results indicate that CDR1as has potential binding sites for these miRNAs (Fig. 8b).

Figure 8 Prediction of CDR1as-related miRNAs binding sites. (a) Predicted target sites of CDR1as-related miRNAs on mRNAs using Targetscan. (b) Binding sites of miRNAs found on CDR1as with the use of RNAhybrid.

\section{Discussion}

Recent studies indicate that muscle development is associated with several coding and non-coding genes, including mRNAs, circular RNAs, and miRNAs [22]. For instance, circFGFR4 induces myoblast differentiation through the upregulation of Wnt3a via the downregulation of miR-107 [56]. Another example includes the inhibition of miR-203 by circSVIL via increasing expression of MEF2C during muscle differentiation [49]. DICER1 is known to play a significant role in vascular smooth muscle development and function by controlling proliferation and contractile differentiation [30]. Therefore studying the transcriptome view of CDR1as and DICER1 in muscle development as well as their relationship during muscle development in SMSCs may contribute to the understanding of genes involved in myogenesis. In this study, we found that CDR1 as and DICER1 contribute significantly to myogenesis through differentiation and are positively correlated.

Based on the properties of coding and non-coding RNAs, various RNAs are known to be related to cancerous diseases $[57,58]$. For instance, circRIP2 promotes bladder cancer progression through miR$1305 / \mathrm{Tfg}-\beta 2 /$ smad3 pathway [59]. Also, miR-135a regulates the proliferation and chemosensitivity of endometrial cancer cells by targeting AKT signaling pathway [60]. However, the number of miRNAs and mRNAs associated with CDR1as and DICER1 in SMSCs remains unknown. In our studies, we knockdown the expression of CDR1as and DICER1 in SMSCs, our interest in the results were based on the downregulated mRNAs and the upregulated miRNAs. A total of 789 mRNAs were differentially expressed, comprising of 401 downregulated mRNAs and 388 upregulated mRNAs. In addition, 27 miRNAs were upregulated, with 16 miRNAs being downregulated after the knockdown of CDR1as in SMSCs. Some of the significantly downregulated mRNAs such as MEF2C [6], ANGPT1 [45], E2F2 [46], CCN1 [47], and FGFR1 [48], are shown to regulate muscle development. Besides, after the knockdown of DICER1, there was a total of 1,113 differentially expressed mRNAs consisting of 686 downregulated mRNAs and 427 upregulated mRNA. Also, there were 15 upregulated miRNAs and 7 downregulated miRNAs. In addition, 
among the significantly downregulated genes such as ANGPT1 [45], MEF2D [41], IGFBP5 [61], E2F2 [46], and CCN1 [47] are also known to be associated with muscle development.

Skeletal muscle development is a gradual process that involves proliferation, differentiation, and fusion. Myoblast differentiation is characterized as the key stage responsible for myogenesis. Moreover, myogenesis is controlled by various signaling regulatory networks, including PI3K-AKT signaling pathway, Focal adhesion, Rap1 signaling pathway, MAPK signaling pathway, and FoxO signaling. For instance, in our study, the KEGG pathway was performed to study the putative functions of DEGs. In siCDR1as profiles, the most enriched pathways of the downregulated mRNAs were (PI3K)-AKT signaling pathway, Focal adhesion, Rap1 signaling pathway, and MAPK signaling pathway. The downregulation of $\mathrm{PI3K} / \mathrm{AKT}$ signaling pathway by miR-106a-5p was shown to inhibit myogenic differentiation of $\mathrm{C} 2 \mathrm{C} 12$ myoblast [62]. Also, according to Jiang et al. the knockdown of PI3-Kinase or its downstream target AKT prevents muscle differentiation in cell culture, while activation of PI3-Kinase and Akt induces myogenic differentiation $[63,64]$. Previous studies have indicated that focal adhesion together with ECM are signaling centers of several intracellular pathways related to cell proliferation and differentiation [65]. Also, focal adhesion plays a significant regulatory role in the biological process including muscle differentiation and striated muscle tissue development [66]. Interestingly, Rap 1 signaling is known to be related beta-adrenergic signaling pathway which has shown to play a crucial role in skeletal muscle growth and development [67]. Moreover, Rap 1 is a small GTPase that regulates different processes, including tightening of cell-cell junction [68], cell polarity, and cell adhesion [69]. MAPK signaling pathway is indicated to regulate the regeneration and proliferation of muscle stem cells [70]. In addition, the MAPK signaling pathway plays a significant role during myoblast differentiation [71, 72]. FoxO signaling pathway is an essential pathway related to muscle development at different stages [66]. Aside from the above-mentioned signaling pathways, the KEGG pathways of the upregulated miRNAs also include WNT and Hippo signaling pathways which are also known to be among the significantly enriched pathways (Fig. 4A). Besides, WNT signaling pathway is involved in the control of satellite cell differentiation and regeneration $[73,74]$, while Hippo signaling pathway is also an essential pathway known to be involved in myogenesis $[75,76]$.

Considering the knockdown of DICER1, we realized that the KEGG pathways of the downregulated mRNAs include PI3K-AKT signaling pathway [62], Focal adhesion [66], and ECM receptor interaction [66], which are also recognized to be associated with muscle development. Moreover, FoxO signaling pathway [66], MAPK signaling pathway [70], Rap1 signaling pathway [68, 69], and mTOR signaling pathway [77] were also known to be associated with the upregulated miRNAs. Moreover, all these pathways are related to muscle development.

According to Xie et al. six elevated miRNAs including miR-1a-3p, miR-133a-3p, miR-133b-3p, miR-206-3p, miR-128-3p, and miR-351-5p were being classified as myogenesis-associated miRNAs (mamiRs), negatively regulated the JNK/MAPK pathway by inhibiting several factors related to the phosphorylation of the JNK/MAPK pathway in skeletal muscle differentiation [78]. miR-146a-5p has been demonstrated to significantly induced VSMC proliferation [55], however, lack of miR-146a did not increase muscular 
dystrophy in mdx mice but was highly expressed in dystrophic muscle [79]. Decreased in the expression of miR-199b-5p induces differentiation of Bone-Marrow Mesenchymal Stem Cells (BMSCs) toward Cardiomyocyte-Like Cells [51]. The Knockdown of miR-199a-3p inhibits slow-to-fast muscle fiber type change in mice and C2C12 Cells [80]. Overexpression of miR-19b-3p prevents VSMC proliferation by targeting CTGF expression [81]. miR-30 family is shown to increase myoblast differentiation in vitro and provide negative feedback on the miRNA pathway [82]. However, according to Zhang et al. miR-30-5p has a negative effect on the differentiation of $\mathrm{C} 2 \mathrm{C} 12$ cells by targeting MBNL [50]. Overexpression of miR3405p is known to reduce the expression of Nrf2 protein in the postexercise skeletal muscle of mice [83]. miR-424 and miR-542-3p are among the most abundant miRNAs in goat muscles [20]. Meanwhile, Garros et al. stated that miR-542 overexpression caused muscle wasting in mice, and reduced mitochondrial function [84]. The knockdown of miR-7 is shown to induce myoblast differentiation [29]. Also, miR-7 is downregulated in skeletal muscle of master athletes [85]. An increase in the expression of miR-7 levels has been recognized as a therapeutic target for opposing muscle dysfunction in DM1 [86]. miR-20a-5p and miR-20b-5p are involved in myoblast differentiation by downregulating the expression of E2F1 [87]. miR-17-5p, miR-378b, miR-199a-5p, and miR-7 may have key roles to play in muscle aging [88], and upregulation of miR-17-5p has been demonstrated to contributes to hypoxia-induced proliferation in human pulmonary artery smooth muscle cells through modulation of p21 and PTEN [52]. Huang et al. stated that miR-374a-5p was shown to negatively regulate MAPK6 expression, and miR-374a-5p may have protective effects against cardiac l/R injury in vivo, and H9C2 H/R injury in vitro [89]. Also, miR-129$5 p$ blocks proliferation of vascular smooth muscle cells (VSMC) by decreasing the expression of Wnt5a [90] and cyclin-dependent kinase 6 (CDK6) cardiac myocytes [91]. Decreased in the expression of miR$27 \mathrm{a} / \mathrm{b}$ resulted in a reduced myoblast proliferation through an increase in myostatin [92]. Overexpression of miR-296 acts as ceRNA for LncRNA CAREL and significantly further inhibits Trp53inp1 and Itm2a to induce proliferation of cardiomyocytes [93]. Overexpression of miR-423-3p in C2C12 myogenic differentiation lead to decreased expression of its target gene Cox6a2 as well as decreased in cellular ATP level [94]. miR-99b-3p is closely related to cancer cell proliferation $[95,96]$. LncRNA UCA1 promotes the progression of cardiac hypertrophy by competitively binding with miR-184 to enhance the expression of HOXA9 [97]. miR-296-3p enhances cardiac differentiation of embryonic stem cells [98]. Moreover, miR128-3p that promotes differentiation of chicken SMSCs [99], was significantly downregulated after the knockdown of DICER1. Based on the reports from different works with regards to the functions of these miRNAs, we can deduce that downregulation of DICER1 increases proliferation but rather decreases differentiation.

In this study, we have made a significant contribution regarding the function of CDR1as and DICER1 in myogenesis. According to the sequencing results, there is a positive correlation between CDR1as and DICER1 differentially expressed miRNAs and mRNAs. Moreover, all the results stated indicates that the knockdown of CDR1as and DICER1 are related to the downregulation of myoblast differentiation. Also, we identified 11 putative miRNAs that can be sponged by CDR1as in SMSCs.

\section{Conclusion}


In summary, we report that CDR1as and DICER1 gene can induce myogenesis in SMSCs through differentiation. Moreover, the knockdown of CDR1as can also decrease the expression levels of DICER1 in SMSCs via vice versa. In future studies, we plan to investigate the functional regulatory pathways of some miRNAs and mRNAs associated with CDR1as and DICER1.

\section{Abbreviations}

circRNAs: circular RNAs; CDR1as: Cerebellar Degeneration-Related protein 1 antisense; miRNAs: microRNAs; SMSCs: goat skeletal muscle satellite cells; ceRNA: Competing Endogenous RNA; mRNAs: messenger RNAs; MEF2: myocyte enhancer factor 2; MEF2C: myocyte enhancer factor 2C; RBPs: RNAbinding proteins; ncRNAs: non-coding RNAs; LD: Longissimus dorsi; DMEM: Dulbecco's modified Eagle's Medium; RIN: RNA Integrity Number; PAGE: Polyacrylamide Gel Electrophoresis; KEGG: Kyoto Encyclopedia of Genes and Genomes; MyoG: myogenin; MEF2D: Myocyte Enhancer Factor 2D; BMP4: Bone Morphogenetic Protein 4; IGFBP5: Insulin-like Growth Factor Binding Protein 5; CCN1: Cysteine-rich 61; and ANGPT1: angiopoietin-1; STAT2: Signal Transducer and Activator of Transcription 2; ICAM1: Intercellular Adhesion Molecule-1; E2F2: E2F transcription factor 2; FGFR1: Fibroblast Growth Factor Receptor 1; ESCC: Esophageal Squamous Cell Carcinoma;

\section{Declarations}

\section{Acknowledgements}

Not applicable

\section{Authors' contributions}

Bismark Kyei and Li Li cultured cells and drafted the manuscript; Liu Yang and Siyuan Zhan reviewed and edited the manuscript; Juntao Li, Yuan Chen, Xujia Zhang, Shuailong Zheng, Xiaoli Xu, Emmanuel Odame, Jiaxue Cao, Dinghui Dai, Jiazhong Guo, Tao Zhong, Linjie Wang analyzed the results; Hongping Zhang supervised the work.

\section{Funding}

This work was supported by the National Natural Science Foundation of China (No. 31772578, 31672402) and the Science and Technology Program of Sichuan Province, China (No. 2016NYZ0045).

\section{Availability of data and materials}

The datasets used and/or analyzed during the current study are available from the corresponding author on reasonable request.

\section{Ethics approval}


This study was conducted in accordance with the Regulations for the Administration of Affairs Concerning Experimental Animals (Ministry of Science and Technology, China) and was approved by the Animal Care and Use Committee of the College of Animal Science and Technology, Sichuan Agricultural University, Sichuan, China.

\section{Consent for publication}

Not applicable

\section{Competing interests}

The authors declare that they have no competing interests.

\section{Author's details}

College of Animal Science and Technology, Sichuan Agricultural University, Chengdu 611130, China.

${ }^{1}$ These authors contributed equally to this work.

\section{References}

1. Molkentin JD, Olson EN. Defining the regulatory networks for muscle development. Curr Opin Genet Dev. 1996; 6(4):445-453.

2. Giordani L, Parisi A, Le Grand F. Chapter Six - Satellite Cell Self-Renewal. In: Current Topics in Developmental Biology. Edited by Sassoon D, vol. 126: Academic Press. 2018; 177-203.

3. ChargÉ SBP, Rudnicki MA. Cellular and Molecular Regulation of Muscle Regeneration. Physiol Rev. 2004; 84(1):209-238.

4. Bentzinger CF, Wang YX, Rudnicki MA. Building muscle: molecular regulation of myogenesis. Cold Spring Harb Perspect Biol. 2012; 4(2):a008342.

5. Fan C-M, Li L, Rozo ME, Lepper C. Making skeletal muscle from progenitor and stem cells: development versus regeneration. Wiley Interdiscip Rev: Dev Biol. 2012; 1(3):315-327.

6. Jama A, Huang D, Alshudukhi AA, Chrast R, Ren H. Lipin1 is required for skeletal muscle development by regulating MEF2C and MyoD expression. J Physiol. 2019; 597(3):889-901.

7. Liu H, Jiang Q, Ju Z, Guan S, He B. Pentraxin 3 promotes cardiac differentiation of mouse embryonic stem cells through JNK signaling pathway. Cell Biol Int. 2018; 42(11):1556-1563.

8. Sahoo S, Meijles DN, Al Ghouleh I, Tandon M, Cifuentes-Pagano E, Sembrat J, Rojas M, Goncharova E, Pagano PJ. MEF2C-MYOCD and Leiomodin1 Suppression by miRNA-214 Promotes Smooth Muscle Cell Phenotype Switching in Pulmonary Arterial Hypertension. Plos one. 2016; 11(5):e0153780-e0153780.

9. Adler K, Molina PE, Simon L. Epigenomic mechanisms of alcohol-induced impaired differentiation of skeletal muscle stem cells; role of Class IIA histone deacetylases. Physiol Genomics. 2019; 
51(9):471-479.

10. Hagan M, Zhou M, Ashraf M, Kim I-M, Su H, Weintraub NL, Tang Y. Long noncoding RNAs and their roles in skeletal muscle fate determination. Noncoding RNA Investig. 2017; 1:24.

11. Apponi LH, Corbett AH, Pavlath GK. RNA-binding proteins and gene regulation in myogenesis. Trends Pharmacol Sci. 2011; 32(11):652-658.

12. Mok GF, Lozano-Velasco E, Münsterberg A. microRNAs in skeletal muscle development. Semin Cell Dev Biol. 2017; 72:67-76.

13. Chen J-F, Mandel EM, Thomson JM, Wu Q, Callis TE, Hammond SM, Conlon FL, Wang D-Z. The role of microRNA-1 and microRNA-133 in skeletal muscle proliferation and differentiation. Nat Genet. 2006; 38(2):228-233.

14. Bartel DP. MicroRNAs: Genomics, Biogenesis, Mechanism, and Function. Cell. 2004; 116(2):281-297.

15. Bernstein E, Kim SY, Carmell MA, Murchison EP, Alcorn H, Li MZ, Mills AA, Elledge SJ, Anderson KV, Hannon GJ. Dicer is essential for mouse development. Nat Genet. 2003; 35(3):215.

16. Yang WJ, Yang DD, Na S, Sandusky GE, Zhang Q, Zhao G. Dicer is required for embryonic angiogenesis during mouse development. J Biol Chem. 2005; 280(10):9330-9335.

17. Özcan S. microRNAs in Pancreatic $\beta$-Cell Physiology. In: microRNA: Basic Science: From Molecular Biology to Clinical Practice. Edited by Santulli G. Cham: Springer International Publishing. 2015; 101117.

18. Goljanek-Whysall K, Sweetman D, Abu-Elmagd M, Chapnik E, Dalmay T, Hornstein E, Münsterberg A. MicroRNA regulation of the paired-box transcription factor Pax3 confers robustness to developmental timing of myogenesis. Proc Natl Acad Sci. 2011; 108(29):11936-11941.

19. Zou C, Li J, Luo W, Li L, Hu A, Fu Y, Hou Y, Li C. Transcriptome analysis reveals long intergenic noncoding RNAs involved in skeletal muscle growth and development in pig. Sci Rep. 2017; 7(1):87048704.

20. Guo J, Zhao W, Zhan S, Li L, Zhong T, Wang L, Dong Y, Zhang H. Identification and Expression Profiling of miRNAome in Goat longissimus dorsi Muscle from Prenatal Stages to a Neonatal Stage. Plos One. 2016; 11(10):e0165764.

21. Wang J, Ren Q, Hua L, Chen J, Zhang J, Bai H, Li H, Xu B, Shi Z, Cao H et al. Comprehensive Analysis of Differentially Expressed mRNA, IncRNA and circRNA and Their ceRNA Networks in the Longissimus Dorsi Muscle of Two Different Pig Breeds. Int J Mol Sci. 2019; 20(5):1107.

22. Das A, Das A, Das D, Abdelmohsen K, Panda AC. Circular RNAs in myogenesis. BBA-Gene Regul Mech. 2019; 194372.

23. Hsiao K-Y, Sun HS, Tsai S-J. Circular RNA - New member of noncoding RNA with novel functions. Exp Biol Med (Maywood). 2017; 242(11):1136-1141.

24. Salzman J: Circular RNA Expression. Its Potential Regulation and Function. Trends Genet. 2016; 32(5):309-316. 
25. Li P, Yang X, Yuan W, Yang C, Zhang X, Han J, Wang J, Deng X, Yang H, Li P et al. CircRNA-Cdr1as Exerts Anti-Oncogenic Functions in Bladder Cancer by Sponging MicroRNA-135a. Cell Physiol Biochem. 2018; 46(4):1606-1616.

26. Sang M, Meng L, Sang Y, Liu S, Ding P, Ju Y, Liu F, Gu L, Lian Y, Li J et al. Circular RNA ciRS-7 accelerates ESCC progression through acting as a miR-876-5p sponge to enhance MAGE-A family expression. Cancer Lett. 2018; 426:37-46.

27. Kyei B, Li L, Yang L, Zhan S, Zhang H. CDR1as/miRNAs-Related Regulatory Mechanisms in Muscle Development and Diseases. Gene. 2020;144315.

28. Piwecka M, Glažar P, Hernandez-Miranda LR, Memczak S, Wolf SA, Rybak-Wolf A, Filipchyk A, Klironomos F, Cerda Jara CA, Fenske $P$ et al. Loss of a mammalian circular RNA locus causes miRNA deregulation and affects brain function. Science. 2017; 357(6357):eaam8526.

29. Li L, Chen Y, Nie L, Ding X, Zhang X, Zhao W, Xu X, Kyei B, Dai D, Zhan S et al. MyoD-induced circular RNA CDR1as promotes myogenic differentiation of skeletal muscle satellite cells. BBA - Gene Regul Mech 2019; 807-821.

30. Albinsson S, Suarez Y, Skoura A, Offermanns S, Miano JM, Sessa WC. MicroRNAs are necessary for vascular smooth muscle growth, differentiation, and function. Arterioscler Thromb Vasc Biol. 2010; 30(6):1118-1126.

31. Park C, Yan W, Ward SM, Hwang SJ, Wu Q, Hatton WJ, Park JK, Sanders KM, Ro S. MicroRNAs Dynamically Remodel Gastrointestinal Smooth Muscle Cells. Plos One. 2011; 6(4):e18628.

32. Zhao W, Chen L, Zhong T, Wang L, Guo J, Dong Y, Feng J, Song T, Li L, Zhang H. The differential proliferation and differentiation ability of skeletal muscle satellite cell in Boer and Nanjiang brown goats. Small Ruminant Res. 2018; 169:99-107.

33. Dong Y, Xie M, Jiang Y, Xiao N, Du X, Zhang W, Tosser-Klopp G, Wang J, Yang S, Liang J et al. Sequencing and automated whole-genome optical mapping of the genome of a domestic goat (Capra hircus). Nat Biotechnol. 2013; 31(2):135-141.

34. Langmead B, Salzberg SL. Fast gapped-read alignment with Bowtie 2. Nat Methods. 2012; 9(4):357359.

35. Langmead B, Trapnell C, Pop M, Salzberg SL. Ultrafast and memory-efficient alignment of short DNA sequences to the human genome. Genome Biol. 2009; 10(3):R25.

36. Kim D, Pertea G, Trapnell C, Pimentel H, Kelley R, Salzberg SL. TopHat2: accurate alignment of transcriptomes in the presence of insertions, deletions and gene fusions. Genome Biol. 2013; 14(4):R36.

37. Trapnell C, Williams BA, Pertea G, Mortazavi A, Kwan G, van Baren MJ, Salzberg SL, Wold BJ, Pachter L. Transcript assembly and quantification by RNA-Seq reveals unannotated transcripts and isoform switching during cell differentiation. Nat Biotechnol. 2010; 28(5):511-515.

38. Xie C, Mao X, Huang J, Ding Y, Wu J, Dong S, Kong L, Gao G, Li C-Y, Wei L. KOBAS 2.0: a web server for annotation and identification of enriched pathways and diseases. Nucleic Acids Res. 2011; 39(suppl_2):W316-W322. 
39. Schmittgen TD, Livak KJ. Analyzing real-time PCR data by the comparative CT method. Nat Protoc. 2008; 3(6):1101-1108.

40. Foulkes WD, Priest JR, Duchaine TF. DICER1: mutations, microRNAs and mechanisms. Nat Rev Cancer. 2014; 14(10):662-672.

41. Davegårdh C, Broholm C, Perfilyev A, Henriksen T, García-Calzón S, Peijs L, Hansen NS, Volkov P, Kjøbsted R, Wojtaszewski JFP et al. Abnormal epigenetic changes during differentiation of human skeletal muscle stem cells from obese subjects. BMC Med. 2017; 15(1):39.

42. Umemoto T, Furutani Y, Murakami M, Matsui T, Funaba M. Endogenous Bmp4 in myoblasts is required for myotube formation in C2C12 cells. BBA - Gen Subjects. 2011; 1810(12):1127-1135.

43. Pegoraro V, Merico A, Angelini C. MyomiRNAs Dysregulation in ALS Rehabilitation. Brain Sci. 2019; $9(1)$.

44. Wang $\mathrm{K}$, Wang $\mathrm{C}$, Xiao $\mathrm{F}$, Wang $\mathrm{H}$, Wu Z. JAK2/STAT2/STAT3 are required for myogenic differentiation. J Biol Chem. 2008; 283(49):34029-34036.

45. Mofarrahi M, McClung JM, Kontos CD, Davis EC, Tappuni B, Moroz N, Pickett AE, Huck L, Harel S, Danialou $\mathrm{G}$ et al. Angiopoietin-1 enhances skeletal muscle regeneration in mice. Am J Physiol-Reg, Integr Comp Physiol. 2015; 308(7):R576-R589.

46. Yan Z, Choi S, Liu X, Zhang M, Schageman JJ, Lee SY, Hart R, Lin L, Thurmond FA, Williams RS. Highly coordinated gene regulation in mouse skeletal muscle regeneration. $J$ Biol Chem. 2003; 278(10):8826-8836.

47. Raissadati A, Nykänen Al, Tuuminen R, Syrjälä So, Krebs R, Arnaudova R, Rouvinen E, Wang X, Poller W, Lemström KB. Systemic overexpression of matricellular protein CCN1 exacerbates obliterative bronchiolitis in mouse tracheal allografts. Transpl Int. 2015; 28(12):1416-1425.

48. Sogos V, Balaci L, Ennas MG, Dell'Era P, Presta M, Gremo F. Developmentally regulated expression and localization of fibroblast growth factor receptors in the human muscle. Deve Dynam. 1998; 211(4):362-373.

49. Ouyang H, Chen X, Li W, Li Z, Nie Q, Zhang X. Circular RNA circSVIL Promotes Myoblast Proliferation and Differentiation by Sponging miR-203 in Chicken. Front Genet. 2018; 9(172).

50. Zhang B-W, Cai H-F, Wei X-F, Sun J-J, Lan X-Y, Lei C-Z, Lin F-P, Qi X-L, Plath M, Chen H. miR-30-5p Regulates Muscle Differentiation and Alternative Splicing of Muscle-Related Genes by Targeting MBNL. Int J Mol Sci. 2016; 17(2).

51. Dai F, Du P, Chang Y, Ji E, Xu Y, Wei C, Li J. Downregulation of MiR-199b-5p Inducing Differentiation of Bone-Marrow Mesenchymal Stem Cells (BMSCs) Toward Cardiomyocyte-Like Cells via HSF1/HSP70 Pathway. Med Sci Monit. 2018; 24:2700-2710.

52. Liu G, Hao P, Xu J, Wang L, Wang Y, Han R, Ying M, Sui S, Liu J, Li X. Upregulation of microRNA-17-5p contributes to hypoxia-induced proliferation in human pulmonary artery smooth muscle cells through modulation of p21 and PTEN. Respir Res. 2018; 19(1):200.

53. Zhang P, Zheng C, Ye H, Teng Y, Zheng B, Yang X, Zhang J. MicroRNA-365 Inhibits Vascular Smooth Muscle Cell Proliferation through Targeting Cyclin D1. Int J Med Sci. 2014; 11(8):765-770. 
54. Elia L, Quintavalle M, Zhang J, Contu R, Cossu L, Latronico MVG, Peterson KL, Indolfi C, Catalucci D, Chen $\mathrm{J}$ et al. The knockout of miR-143 and -145 alters smooth muscle cell maintenance and vascular homeostasis in mice: correlates with human disease. Cell Death \& Differ. 2009; 16(12):1590-1598.

55. Xue L, Luo S, Ding H, Liu Y, Huang W, Fan X, Wu M, Jian X, Huang C, Luo J et al. Upregulation of miR$146 a-5 p$ is associated with increased proliferation and migration of vascular smooth muscle cells in aortic dissection. J Clin Lab Anal. 2019; 33(4):e22843.

56. Li H, Wei X, Yang J, Dong D, Hao D, Huang Y, Lan X, Plath M, Lei C, Ma Y. circFGFR4 promotes differentiation of myoblasts via binding miR-107 to relieve its inhibition of Wnt3a. Mol Ther-Nucleic Acids. 2018; 11:272-283.

57. Di Leva G, Garofalo M, Croce CM. MicroRNAs in Cancer. Annu Rev Pathol: Mech. 2014; 9(1):287-314.

58. Wu P, Mo Y, Peng M, Tang T, Zhong Y, Deng X, Xiong F, Guo C, Wu X, Li Y et al: Emerging role of tumorrelated functional peptides encoded by IncRNA and circRNA. Mol Cancer. 2020; 19(1):22.

59. Su Y, Feng W, Shi J, Chen L, Huang J, Lin T. circRIP2 accelerates bladder cancer progression via miR1305/Tgf- $\beta 2 /$ smad3 pathway. Mol Cancer. 2020; 19(1):23.

60. Wang J, Zhang L, Jiang W, Zhang R, Zhang B, Silayiding A, Duan X. MicroRNA-135a promotes proliferation, migration, invasion and induces chemoresistance of endometrial cancer cells. Eur $\mathrm{J}$ Obstet Gyn R B: X. 2020; 5:100103.

61. Zhang WR, Zhang HN, Wang YM, Dai Y, Liu XF, Li X, Ding XB, Guo H. miR-143 regulates proliferation and differentiation of bovine skeletal muscle satellite cells by targeting IGFBP5. In Vitro Cell Dev - An. 2017; 53(3):265-271.

62. Li X, Zhu Y, Zhang H, Ma G, Wu G, Xiang A, Shi XE, Yang GS, Sun S. MicroRNA-106a-5p Inhibited C2C12 Myogenesis via Targeting PIK3R1 and Modulating the PI3K/AKT Signaling. Genes (Basel). 2018; 9(7):333.

63. Jiang B-H, Zheng JZ, Vogt PK. An essential role of phosphatidylinositol 3-kinase in myogenic differentiation. Proc Natl Acad Sci. 1998; 95(24):14179.

64. Jiang B-H, Aoki M, Zheng JZ, Li J, Vogt PK. Myogenic signaling of phosphatidylinositol 3-kinase requires the serine-threonine kinase Akt/protein kinase B. Proc Natl Acad Sci. 1999; 96(5):2077.

65. Romer LH, Birukov KG, Garcia JGN. Focal adhesions: paradigm for a signaling nexus. Circ Res. 2006. 98(5):606-616.

66. Li Y, Chen Y, Jin W, Fu S, Li D, Zhang Y, Sun G, Jiang R, Han R, Li Z et al. Analyses of MicroRNA and mRNA Expression Profiles Reveal the Crucial Interaction Networks and Pathways for Regulation of Chicken Breast Muscle Development. Front Genet. 2019; 10:197-197.

67. Lynch GS, Ryall JG. Role of $\beta$-Adrenoceptor Signaling in Skeletal Muscle: Implications for Muscle Wasting and Disease. Physiol Rev. 2008; 88(2):729-767.

68. Kooistra MRH, Dubé N, Bos JL. Rap1: a key regulator in cell-cell junction formation. J Cell Sci. 2007; 120(1):17. 
69. Boettner B, Van Aelst L. Control of cell adhesion dynamics by Rap1 signaling. Curr Opin Cell Biol. 2009; 21(5):684-693.

70. Ling Y, Zheng Q, Sui M, Zhu L, Xu L, Zhang Y, Liu Y, Fang F, Chu M, Ma Y et al. Comprehensive Analysis of LncRNA Reveals the Temporal-Specific Module of Goat Skeletal Muscle Development. Int J Mol Sci. 2019; 20(16):3950.

71. Aboalola D, Han VKM. Insulin-Like Growth Factor Binding Protein-6 Promotes the Differentiation of Placental Mesenchymal Stem Cells into Skeletal Muscle Independent of Insulin-Like Growth Factor Receptor-1 and Insulin Receptor. Stem Cells Int. 2019;9245938-9245938.

72. Gonzalez I, Tripathi G, Carter EJ, Cobb LJ, Salih DAM, Lovett FA, Holding C, Pell JM. Akt2, a novel functional link between p38 mitogen-activated protein kinase and phosphatidylinositol 3-kinase pathways in myogenesis. Mol Cell Biol. 2004; 24(9):3607-3622.

73. Brack AS, Conboy IM, Conboy MJ, Shen J, Rando TA. A Temporal Switch from Notch to Wnt Signaling in Muscle Stem Cells Is Necessary for Normal Adult Myogenesis. Cell Stem Cell. 2008; 2(1):50-59.

74. Ferrari L, Bragato C, Brioschi L, Spreafico M, Esposito S, Pezzotta A, Pizzetti F, Moreno-Fortuny A, Bellipanni G, Giordano A et al. HDAC8 regulates canonical Wnt pathway to promote differentiation in skeletal muscles. J CelL Physiol. 2019; 234(5):6067-6076.

75. Watt KI, Goodman CA, Hornberger TA, Gregorevic P. The Hippo Signaling Pathway in the Regulation of Skeletal Muscle Mass and Function. Exerc Sport Sci Rev. 2018; 46(2):92-96.

76. Sun C, De Mello V, Mohamed A, Ortuste Quiroga HP, Garcia-Munoz A, Al Bloshi A, Tremblay AM, von Kriegsheim A, Collie-Duguid E, Vargesson $\mathrm{N}$ et al. Common and Distinctive Functions of the Hippo Effectors Taz and Yap in Skeletal Muscle Stem Cell Function. Stem Cells. 2017; 35(8):1958-1972.

77. Ge Y, Sun Y, Chen J. IGF-II is regulated by microRNA-125b in skeletal myogenesis. The J cell biol. 2011; 192(1):69-81.

78. Xie S-J, Li J-H, Chen H-F, Tan Y-Y, Liu S-R, Zhang Y, Xu H, Yang J-H, Liu S, Zheng L-L et al. Inhibition of the JNK/MAPK signaling pathway by myogenesis-associated miRNAs is required for skeletal muscle development. Cell Death \& Differ. 2018; 25(9):1581-1597.

79. Bronisz-Budzyńska I, Chwalenia K, Mucha O, Podkalicka P, Karolina Bukowska S, Józkowicz A, Łoboda A, Kozakowska M, Dulak J. miR-146a deficiency does not aggravate muscular dystrophy in mdx mice. Skeletal Muscle. 2019; 9(1):22.

80. Zhang Y, Yang M, Zhou P, Yan H, Zhang Z, Zhang H, Qi R, Liu J. $\beta$-Hydroxy- $\beta$-methylbutyrate-Induced Upregulation of miR-199a-3p Contributes to Slow-To-Fast Muscle Fiber Type Conversion in Mice and C2C12 Cells. J Agric Food Chem. 2020; 68(2):530-540.

81. Wang W-B, Li H-P, Yan J, Zhuang F, Bao M, Liu J-T, Qi Y-X, Han Y. CTGF regulates cyclic stretchinduced vascular smooth muscle cell proliferation via microRNA-19b-3p. Exp Cell Res. 2019; 376(1):77-85.

82. Guess MG, Barthel KKB, Harrison BC, Leinwand LA. miR-30 Family microRNAs Regulate Myogenic Differentiation and Provide Negative Feedback on the microRNA Pathway. Plos One. 2015; 
10(2):e0118229.

83. T M, Y L, J W, Y Z. miR-340-5p: A potential direct regulator of Nrf2 expression in the post-exercise skeletal muscle of mice. Mol Med Rep. 2019; 19(2):1340-1348.

84. Garros RF, Paul R, Connolly M, Lewis A, Garfield BE, Natanek SA, Bloch S, Mouly V, Griffiths MJ, Polkey MI et al. MicroRNA-542 Promotes Mitochondrial Dysfunction and SMAD Activity and Is Elevated in Intensive Care Unit-acquired Weakness. Am J Respir Crit Care Med. 2017; 196(11):14221433.

85. Koltai E, Bori Z, Osvath P, Ihasz F, Peter S, Toth G, Degens H, Rittweger J, Boldogh I, Radak Z. Master athletes have higher miR-7, SIRT3 and SOD2 expression in skeletal muscle than age-matched sedentary controls. Redox Biol. 2018; 19:46-51.

86. Sabater-Arcis M, Bargiela A, Furling D, Artero R. miR-7 Restores Phenotypes in Myotonic Dystrophy Muscle Cells by Repressing Hyperactivated Autophagy. Mol Ther - Nucleic Acids. 2020; 19:278-292.

87. Luo W, Li G, Yi Z, Nie Q, Zhang X. E2F1-miR-20a-5p/20b-5p auto-regulatory feedback loop involved in myoblast proliferation and differentiation. Sci Rep. 2016; 6(1):27904.

88. Jia B, Liu Y, Li Q, Zhang J, Ge C, Wang G, Chen G, Liu D, Yang F. Altered miRNA and mRNA Expression in Sika Deer Skeletal Muscle with Age. Genes (Basel). 2020; 11(2).

89. Huang Z-Q, Xu W, Wu J-L, Lu X, Chen X-M. MicroRNA-374a protects against myocardial ischemiareperfusion injury in mice by targeting the MAPK6 pathway. Life Sci. 2019; 232:116619.

90. Zhang Y, Liu Z, Zhou M, Liu C. MicroRNA-129-5p inhibits vascular smooth muscle cell proliferation by targeting Wnt5a. Exp Ther Med. 2016; 12(4):2651-2656.

91. Majumdar G, Raghow R. Trichostatin A induces a unique set of microRNAs including miR-129-5p that blocks cyclin-dependent kinase 6 expression and proliferation in $\mathrm{H} 9 \mathrm{c} 2$ cardiac myocytes. Mol Cell Biochem. 2016; 415(1):39-49.

92. McFarlane C, Vajjala A, Arigela H, Lokireddy S, Ge X, Bonala S, Manickam R, Kambadur R, Sharma M. Negative Auto-Regulation of Myostatin Expression is Mediated by Smad3 and MicroRNA-27. Plos One. 2014; 9(1):e87687.

93. Cai B, Ma W, Ding F, Zhang L, Huang Q, Wang X, Hua B, Xu J, Li J, Bi C et al. The Long Noncoding RNA CAREL Controls Cardiac Regeneration. J Am Coll Cardiol. 2018; 72(5):534-550.

94. Siengdee P, Trakooljul N, Murani E, Schwerin M, Wimmers K, Ponsuksili S. MicroRNAs Regulate Cellular ATP Levels by Targeting Mitochondrial Energy Metabolism Genes during C2C12 Myoblast Differentiation. Plos One. 2015; 10(5):e0127850.

95. Chang Se, Gao Z, Yang Y, He K, Wang X, Wang L, Gao N, Li H, He X, Huang C. miR-99b-3p is induced by vitamin $\mathrm{D} 3$ and contributes to its antiproliferative effects in gastric cancer cells by targeting HoxD3. In: Biol Chem. vol. 400. 2019; 1079.

96. Yao X, Zhang H, Liu Y, Liu X, Wang X, Sun X, Cheng Y. miR-99b-3p promotes hepatocellular carcinoma metastasis and proliferation by targeting protocadherin 19. Gene. 2019; 698:141-149. 
97. Zhou G, Li C, Feng J, Zhang J, Fang Y. IncRNA UCA1 Is a Novel Regulator in Cardiomyocyte Hypertrophy through Targeting the miR-184/HOXA9 Axis. Cardiorenal Med. 2018; 8(2):130-139.

98. Sun $M$, Yan $X$, Bian $Y$, Caggiano AO, Morgan JP. Improving murine embryonic stem cell differentiation into cardiomyocytes with neuregulin-1: differential expression of microRNA. Am J Physiol-Cell Physiol. 2011; 301(1):C21-C30.

99. Shen X, Liu Z, Cao X, He H, Han S, Chen Y, Cui C, Zhao J, Li D, Wang Y et al. Circular RNA profiling identified an abundant circular RNA circTMTC1 that inhibits chicken skeletal muscle satellite cell differentiation by sponging miR-128-3p. Int J Biol Sci. 2019; 15(10):2265-2281.

\section{Tables}

Table 1 Summary of reads from raw data and clean read for mRNAs (siCDR1as)

\begin{tabular}{lccc}
\hline Sample & Raw Reads & Clean Reads & Clean Reads (\%) \\
\hline siCDR1as_1 & 51865796 & 51084670 & $98.49 \%$ \\
siCDR1as_2 & 64928768 & 63448702 & $97.72 \%$ \\
siCDR1as_3 & 62668742 & 61124018 & $97.53 \%$ \\
siNC_1 & 71050762 & 69247162 & $97.46 \%$ \\
siNC_2 & 60328494 & 58720344 & $97.33 \%$ \\
siNC_3 & 73310062 & 70808056 & $96.59 \%$ \\
\hline
\end{tabular}

Table 2 Summary of reads from raw data and clean read for miRNAs (siCDR1as)

\begin{tabular}{lccc}
\hline Sample & Raw Reads & Clean Reads & Clean Reads (\%) \\
\hline siCDR1as_1 & 15249256 & 14876516 & $97.56 \%$ \\
siCDR1as_2 & 15109243 & 14776717 & $97.80 \%$ \\
siCDR1as_3 & 16302005 & 16044248 & $98.42 \%$ \\
siNC_1 & 17583292 & 17101617 & $97.26 \%$ \\
siNC_2 & 15392007 & 15172466 & $98.57 \%$ \\
siNC_3 & 16631937 & 16245764 & $97.68 \%$ \\
\hline
\end{tabular}

Table 3 Summary of reads from raw data and clean read for mRNAs (siDICER1) 


$\begin{array}{lccc}\text { SiDICER1_1 } & 114799120 & 107162908 & 93.35 \% \\ \text { siDICER1_2 } & 116729108 & 109896996 & 94.15 \% \\ \text { siDICER1_3 } & 154284884 & 145845612 & 94.53 \% \\ \text { siNC_1 } & 112293404 & 105431736 & 93.89 \% \\ \text { siNC_2 } & 115938944 & 106717500 & 92.05 \% \\ \text { siNC_3 } & 146546000 & 139174744 & 94.97 \%\end{array}$

Table 4 Summary of reads from raw data and clean read for miRNAs (siDICER1)

\begin{tabular}{lccl}
\hline Sample & Raw Reads & Clean Reads & Clean Reads (\%) \\
\hline siDICER1_1 & 13243248 & 12950668 & $97.79 \%$ \\
siDICER1_2 & 16224384 & 15945782 & $98.28 \%$ \\
siDICER1_3 & 13313653 & 13189595 & $99.07 \%$ \\
siNC_1 & 15131051 & 14880303 & $98.34 \%$ \\
siNC_2 & 15827821 & 15533815 & $98.14 \%$ \\
siNC_3 & 12748120 & 12389599 & $97.19 \%$ \\
\hline
\end{tabular}

Table 529 miRNAs that are critical for muscle cells after inhibition of DICER1

miRNAs siDICER1_readcount siNC_readcount Log2|FoldChange| pval padj




\begin{tabular}{|c|c|c|c|c|c|}
\hline miR-340-5p & 4170.709921 & 1334.718 & 7775 & 1.6442 & $E-17$ \\
\hline miR-1 & 2399.157854 & 795.3784952 & 1.5927 & $4.68 \mathrm{E}-13$ & $6.79 \mathrm{E}-11$ \\
\hline miR-199b-5p & 4978.537584 & 2343.874937 & 1.0859 & $1.66 \mathrm{E}-08$ & 1.61E-06 \\
\hline miR-206 & 10463.34296 & 4339.513679 & 1.2698 & $6.98 \mathrm{E}-08$ & $4.05 \mathrm{E}-06$ \\
\hline $\operatorname{miR}-3431-3 p$ & 747.5581005 & 349.0385672 & 1.0997 & 1.93E-05 & 0.00093151 \\
\hline $\operatorname{miR}-133 b$ & 127.0395034 & 39.85125436 & 1.6797 & $7.46 \mathrm{E}-05$ & 0.0027679 \\
\hline miR-542-3p & 1382.692995 & 870.6786347 & 0.66819 & 7.64E-05 & 0.0027679 \\
\hline miR-133a-3p & 291.1553908 & 103.0553655 & 1.5004 & $9.86 \mathrm{E}-05$ & 0.0030471 \\
\hline $\operatorname{miR}-19 b-3 p$ & 1869.320577 & 1147.853935 & 0.70245 & 0.00019374 & 0.004682 \\
\hline miR-30b-5p & 1626.351315 & 1086.047394 & 0.58234 & 0.0005161 & 0.010691 \\
\hline miR-146a & 123.3477915 & 62.44958905 & 0.97531 & 0.0014387 & 0.026076 \\
\hline miR-335-5p & 475.628044 & 306.2321444 & 0.63337 & 0.0017979 & 0.030671 \\
\hline miR-30e-5p & 3605.104766 & 2553.345384 & 0.49672 & 0.0022368 & 0.032433 \\
\hline $\operatorname{miR}-424-5 p$ & 228.9440715 & 124.8912472 & 0.87721 & 0.0036705 & 0.048384 \\
\hline $\operatorname{miR}-215-5 p$ & 30.91083813 & 125.6754952 & -2.0179 & $5.24 \mathrm{E}-08$ & $3.80 \mathrm{E}-06$ \\
\hline $\operatorname{miR}-27 a-5 p$ & 581.7830669 & 1064.597836 & -0.87221 & 0.00010507 & 0.0030471 \\
\hline miR-1307-3p & 1532.225721 & 2164.944693 & -0.49752 & 0.00043459 & 0.0096947 \\
\hline miR-129-5p & 1281.977708 & 1956.16048 & -0.60988 & 0.001126 & 0.02177 \\
\hline miR-128-3p & 3499.573083 & 5393.484712 & -0.62359 & 0.0021869 & 0.032433 \\
\hline miR-296-3p & 130.4572684 & 220.0652404 & -0.74886 & 0.0020456 & 0.032433 \\
\hline $\operatorname{miR}-27 b-5 p$ & 251.479739 & 358.1369536 & -0.50923 & 0.0024619 & 0.033998 \\
\hline miR-7-5p & 25521.84767 & 17040.0976 & 0.58269 & 0.013929 & 0.1122 \\
\hline miR-20a-5p & 6449.065249 & 4617.367179 & 0.48193 & 0.023613 & 0.16304 \\
\hline miR-30a-5p & 4233.470356 & 2966.995856 & 0.51206 & 0.0059082 & 0.063458 \\
\hline miR-17-5p & 3972.513323 & 2800.868924 & 0.50418 & 0.037555 & 0.21782 \\
\hline miR-374a-5p & 2063.02369 & 1275.097278 & 0.69402 & 0.007115 & 0.068542 \\
\hline miR-423-3p & 5150.221875 & 6904.915616 & -0.42311 & 0.0057131 & 0.063458 \\
\hline miR-99b-3p & 285.7822278 & 410.4920085 & -0.52437 & 0.011012 & 0.096768 \\
\hline miR-184 & 127.8900981 & 194.9299834 & -0.60921 & 0.0045266 & 0.029837 \\
\hline
\end{tabular}

Table 643 miRNAs that are critical for muscle cells after inhibition of CDR1 as miRNAs siCDR1as_readcount siNC_readcount Log2|FoldChange| pval padj 


\begin{tabular}{|c|c|c|c|c|c|}
\hline chi-m & 3001.248 & 1810.237 & 0.72912 & $2.60 \mathrm{E}-08$ & $3.12 \mathrm{E}-06$ \\
\hline chi-miR-20a-5p & 7199.622 & 4354.877 & 0.72503 & $2.78 \mathrm{E}-07$ & 2.23E-05 \\
\hline novel_52 & 1128.806 & 613.921 & 0.87711 & $4.69 \mathrm{E}-07$ & 2.82E-05 \\
\hline chi-miR-140-5p & 2204.859 & 1439.661 & 0.61501 & $5.54 \mathrm{E}-06$ & 0.000133 \\
\hline chi-miR-222-3p & 9959.478 & 6640.695 & 0.58454 & $3.94 \mathrm{E}-06$ & 0.000133 \\
\hline chi-let-7i-3p & 367.0999 & 223.6484 & 0.71562 & $9.32 \mathrm{E}-06$ & 0.000186 \\
\hline chi-miR-26a-5p & 84563.05 & 57668.11 & 0.55225 & 8.88E-06 & 0.000186 \\
\hline chi-miR-19a & 348.086 & 134.4014 & 1.3685 & $1.24 \mathrm{E}-05$ & 0.000229 \\
\hline chi-miR-191-5p & 6879.281 & 4573.587 & 0.58895 & 3.03E-05 & 0.000519 \\
\hline chi-miR-362-5p & 764.6337 & 449.3501 & 0.76461 & $3.87 \mathrm{E}-05$ & 0.000619 \\
\hline chi-miR-18a-5p & 246.8867 & 144.2109 & 0.77112 & 0.000187 & 0.002495 \\
\hline chi-miR-15a-5p & 359.2752 & 210.8023 & 0.76674 & 0.00126 & 0.014397 \\
\hline chi-miR-125a-5p & 5395.456 & 3275.853 & 0.71978 & 0.001574 & 0.015266 \\
\hline chi-miR-125b-5p & 62585.56 & 44942.4 & 0.47776 & 0.001648 & 0.015266 \\
\hline chi-miR-29a-3p & 24037.27 & 18357.92 & 0.38886 & 0.001478 & 0.015266 \\
\hline novel_78 & 296.5034 & 198.6685 & 0.57919 & 0.001681 & 0.015266 \\
\hline chi-miR-374b-5p & 5091.916 & 3688.704 & 0.46489 & 0.001843 & 0.015304 \\
\hline novel_31 & 1233.08 & 848.6147 & 0.54022 & 0.002001 & 0.016005 \\
\hline chi-miR-93-5p & 8689.138 & 6836.243 & 0.3461 & 0.002745 & 0.021251 \\
\hline chi-miR-30e-5p & 1262 & 339 & 0.4 & 41 & 061 \\
\hline chi-miR-199a-5p & 68943.3 & 50479.2 & 0.44975 & 0.003504 & 0.025483 \\
\hline chi-miR-365-3p & 1554.539 & 994.1919 & 0.64332 & 0.003964 & 0.027983 \\
\hline chi-miR-181b-5p & 639.2447 & 480.2985 & 0.41174 & 0.004109 & 0.028179 \\
\hline chi-miR-874-3p & 65.97 & 35.7 & 0.8767 & 04 & 85 \\
\hline chi-miR-146a & 1106.627 & 862.9648 & 0.3581 & 0.005852 & 0.035113 \\
\hline chi-miR-30f-5p & 235.0536 & 167.7368 & 0.49004 & 0.007716 & 0.044094 \\
\hline chi-miR-107-3p & 1138.959 & 855.4164 & 0.4132 & 0.008342 & 0.04656 \\
\hline chi-miR-151-3p & 19688.77 & 37772.71 & -0.93987 & $3.45 \mathrm{E}-10$ & $8.29 \mathrm{E}-08$ \\
\hline chi-miR-140-3p & 11045.99 & 15935.61 & -0.52868 & $2.44 \mathrm{E}-06$ & $9.75 \mathrm{E}-05$ \\
\hline chi-miR-379-5p & 2243.846 & 3453.597 & -0.6214 & $2.20 \mathrm{E}-06$ & 9.75E-05 \\
\hline chi-miR-143-3p & 1027419 & 1649770 & -0.68324 & $5.35 \mathrm{E}-06$ & 0.000133 \\
\hline chi-miR-411a-5p & 2529.26 & 4076.524 & -0.68856 & $5.14 \mathrm{E}-06$ & 0.000133 \\
\hline chi-miR-148a-3p & 224034.2 & 369598 & -0.72223 & 9.33E-05 & 0.0014 \\
\hline chi-miR-493-3p & 273.7732 & 430.8949 & -0.64994 & 0.000111 & 0.00156 \\
\hline novel_48 & 685.3696 & 1041.922 & -0.60186 & 0.000232 & 0.002927 \\
\hline chi-miR-1388-5p & 397.6739 & 564.416 & -0.50206 & 0.000924 & 0.011083 \\
\hline chi-miR-218 & 563.6718 & 863.5813 & -0.6145 & 0.001718 & 0.015266 \\
\hline chi-miR-27a-5p & 434.6935 & 594.9322 & -0.4532 & 0.001509 & 0.015266 \\
\hline chi-miR-99b-3p & 251.3425 & 374.3879 & -0.56933 & 0.001849 & 0.015304 \\
\hline chi-miR-184 & 127.8901 & 194.93 & -0.60921 & 0.004527 & 0.029837 \\
\hline chi-miR-708-3p & 446.1509 & 612.6852 & -0.45456 & 0.0046 & 0.029837 \\
\hline chi-miR-24-3p & 59250.53 & 76802.59 & -0.37432 & 0.005523 & 0.033985 \\
\hline chi-miR-106b-3p & 1026.171 & 1415.912 & -0.46227 & 0.006238 & 0.036516 \\
\hline
\end{tabular}

\section{Supplementary Information}


Supplemetal Figure 1 and 2: The expression level of CDR1as and DICER1 in C2C12 transfected with siCDR1as or siDICER1 was validated by qPCR.

Supplemetal Figure 3: KEGG of the Upregulated mRNAs with the top 20 enrichment. Bubble color and size correspond to the $\mathrm{Q}$ value and gene number enriched in the pathway. The rich factor indicates the ratio of the number of DEGs mapped to a certain pathway to the total number of genes mapped to this pathway.

Supplemetal Figure 4: KEGG analysis of the downregulated miRNA-mRNA network. Bubble color and size correspond to the $\mathrm{Q}$ value and gene number enriched in the pathway. The rich factor indicates the ratio of the number of DEGs mapped to a certain pathway to the total number of genes mapped to this pathway.

Supplemetal Figure 5: KEGG of the upregulated mRNAs with the top 20 enrichment. Bubble color and size correspond to the $\mathrm{Q}$ value and gene number enriched in the pathway. The rich factor indicates the ratio of the number of DEGs mapped to a certain pathway to the total number of genes mapped to this pathway.

Supplemetal Figure 6: KEGG analysis of downregulated miRNAs targets mRNAs. Bubble color and size correspond to the $\mathrm{Q}$ value and gene number enriched in the pathway. The rich factor indicates the ratio of the number of DEGs mapped to a certain pathway to the total number of genes mapped to this pathway.

Supplemetal Figure 7: KEGG analysis of overlapped upregulated mRNAs. Bubble color and size correspond to the $\mathrm{Q}$ value and gene number enriched in the pathway. The rich factor indicates the ratio of the number of DEGs mapped to a certain pathway to the total number of genes mapped to this pathway.

\section{Figures}

A
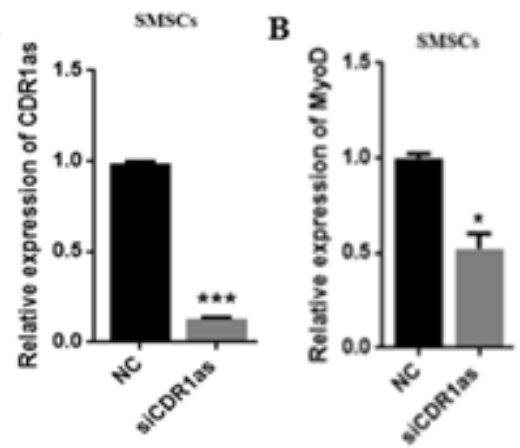

C
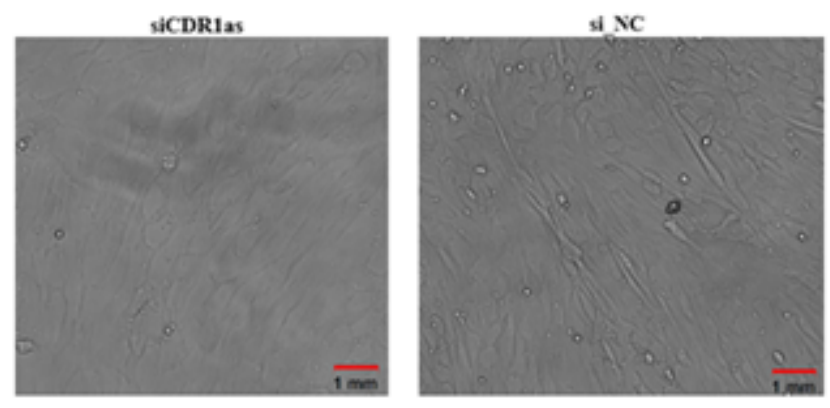

D
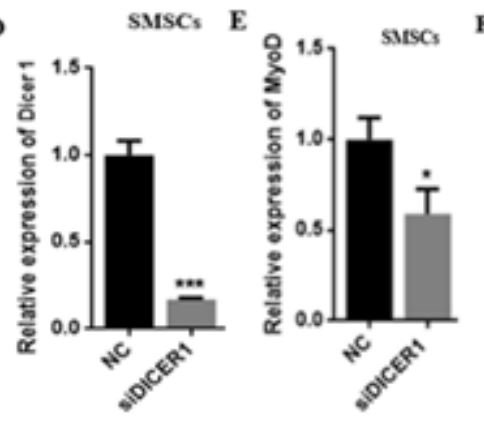

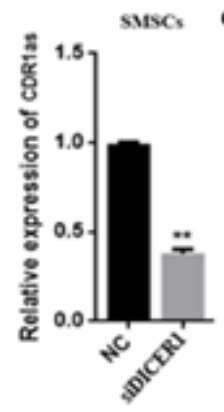

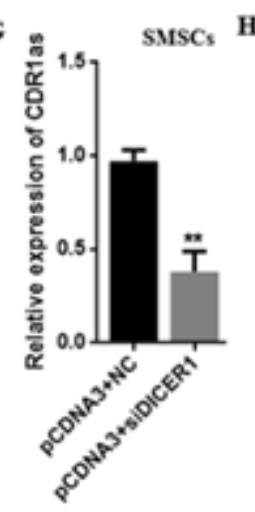

ADICERI
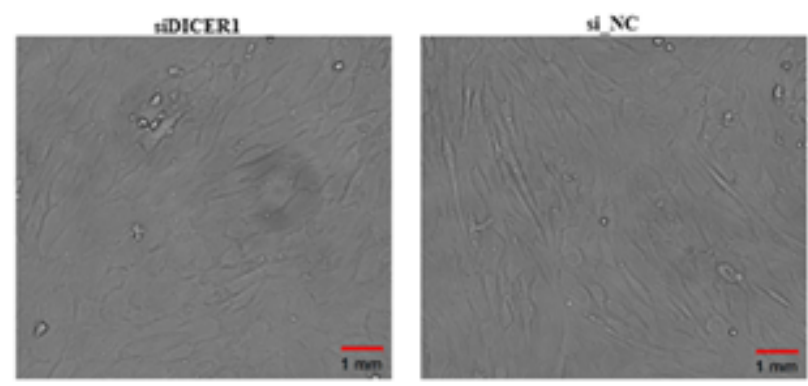
Figure 1

The Expression of Cerebellar Degeneration-Related protein 1 antisense (CDR1as) and DICER1 on skeletal muscle satellite cells (SMSCs) differentiation. ( $a$ and $d$ ) The expression level of CDR1as and DICER1 in SMSCs transfected with siCDR1 as or siDICER1 was validated. (b and e) QPCR analysis of MyoD expression in SMSCs after transfection with siCDR1as or negative control (NC) and siDICER1 or NC. (C and $h$ ) A representative of microscopic images after the knockdown of CDR1 as and DICER1. ( $f$ and $g$ ) SMSCs were transfected with NC or siDICER1, pCDNA3+NC or pCDNA3+siDICER1 to determine the relationship between CDR1 as and DICER1. The $p$ values were analyzed by Student's test; $p<0.05$.

A Pearson correlation between samples (mRNAs-siCDRlas)

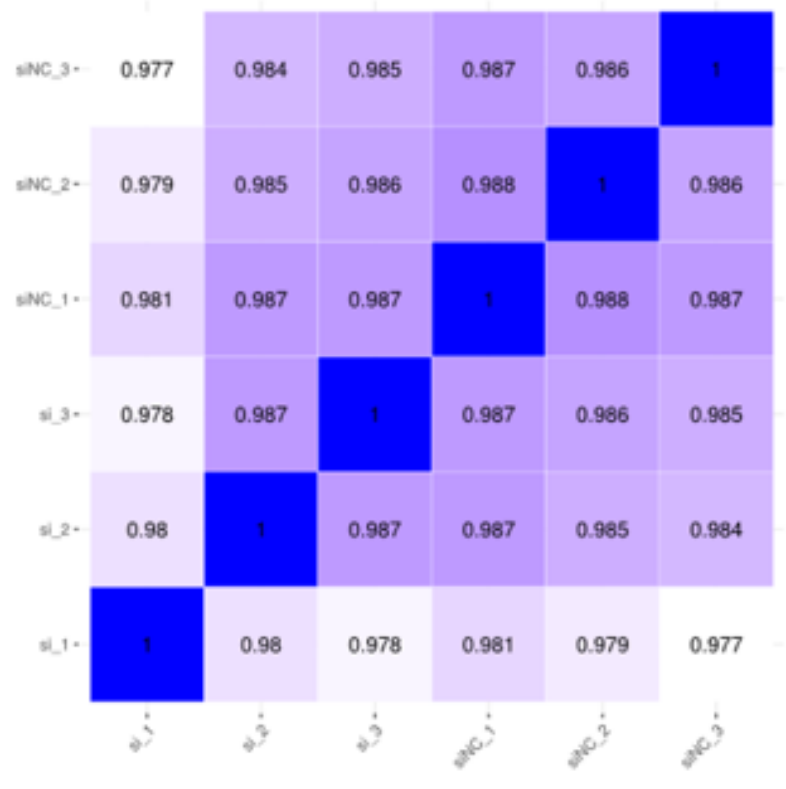

C Pearson corrèlation between samples (mRNAs-siDICERI)

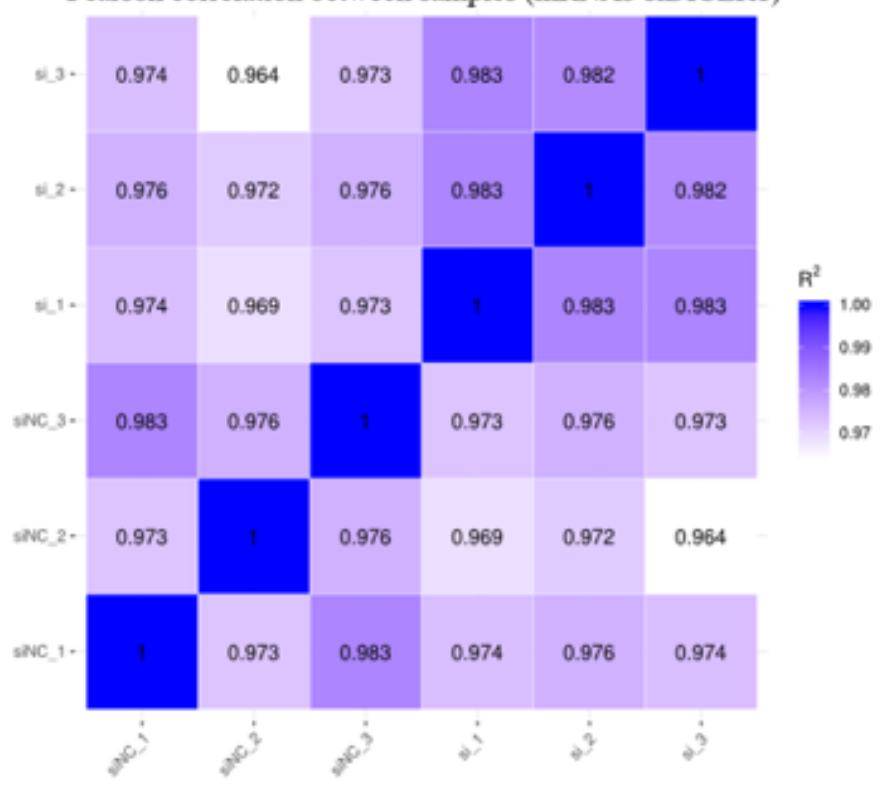

B Pearson correlation between samples (miRNAs-siCDR1as)

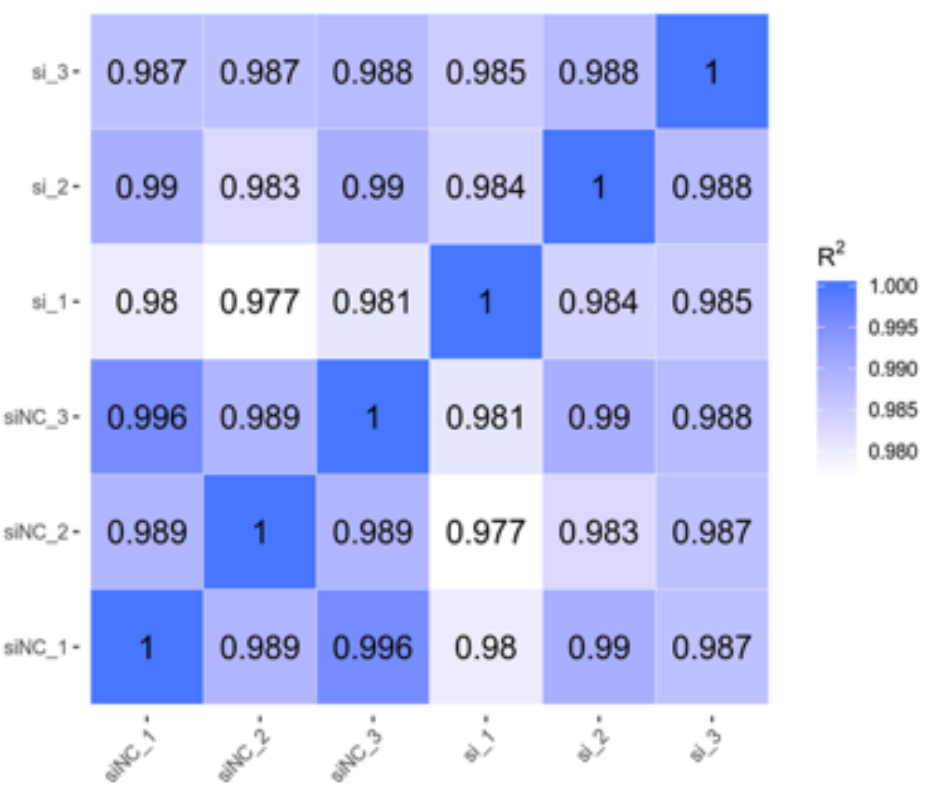

D

Pearson correlation between samples (miRNAs-siDICER1)

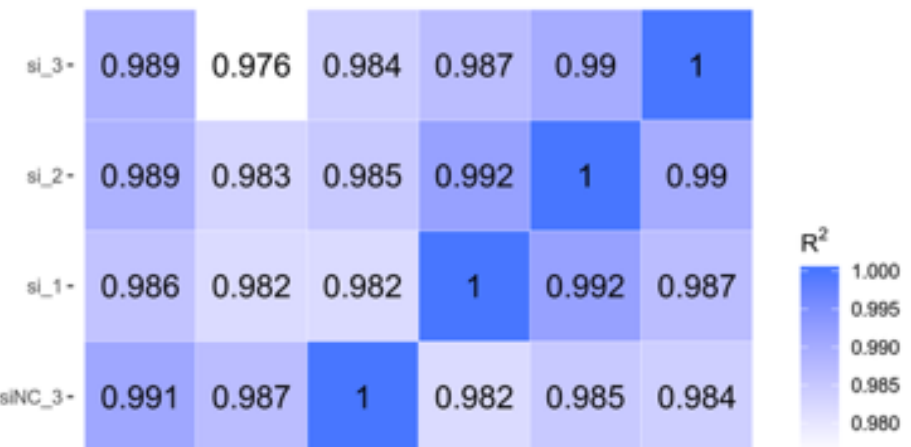

\begin{tabular}{l|l|l|l|l|l|l} 
sinc_2- & 0.986 & 1 & 0.987 & 0.982 & 0.983 & 0.976
\end{tabular}

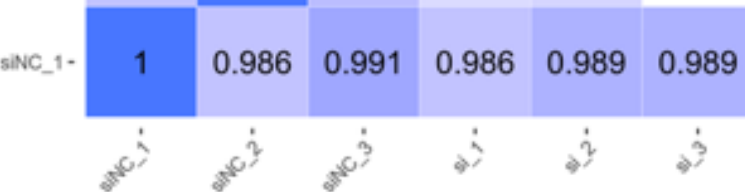

Figure 2 
siCDR1as and siDICER1 Pearson correlation chart. (a-d) The abscissa and the ordinate were the respective samples, and the abscissa and the ordinate of each patch represented the correlation of siCDR1as and siDICER1 samples. Importantly, two completely related genomes had a value of 1 . The closer to 1 the relative value is, the larger the Pearson correlation coefficient (PCC) for the siCDR1as and siDICER1 samples; conversely, the closer to 0 the relative value was, the smaller the PCC between the siCDR1as and siDICER1 samples.

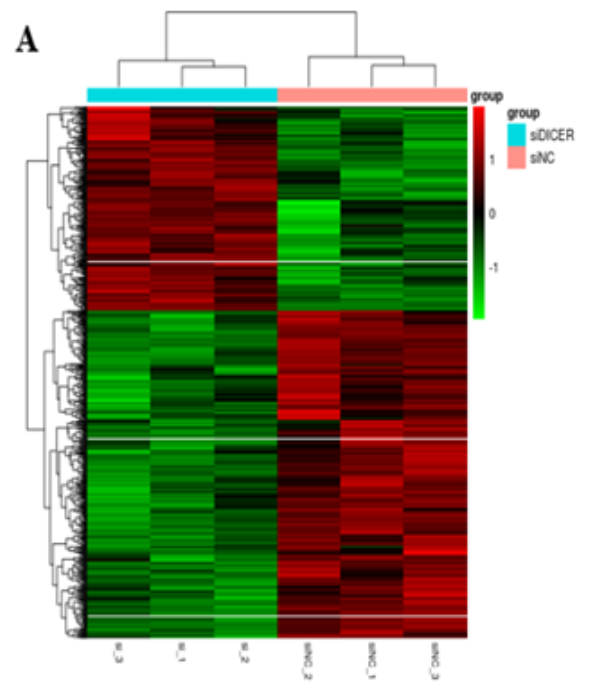

B

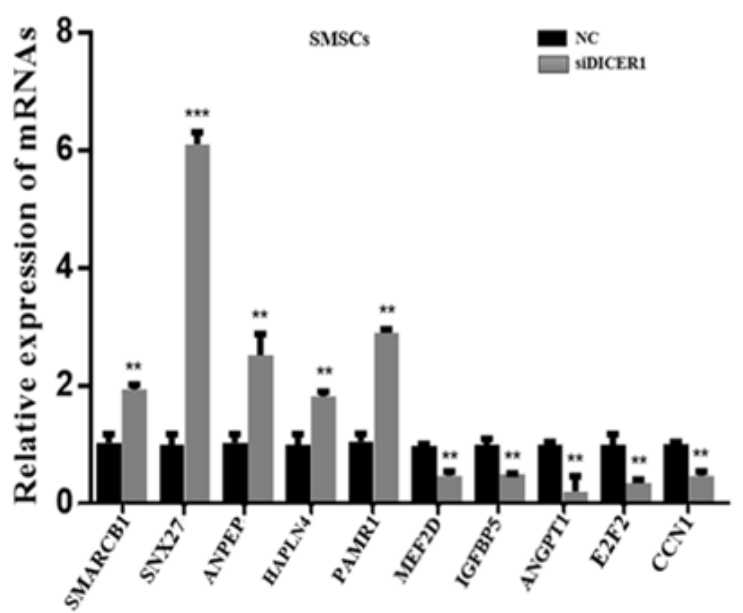

C

Top 20 of KEGG Enrichment

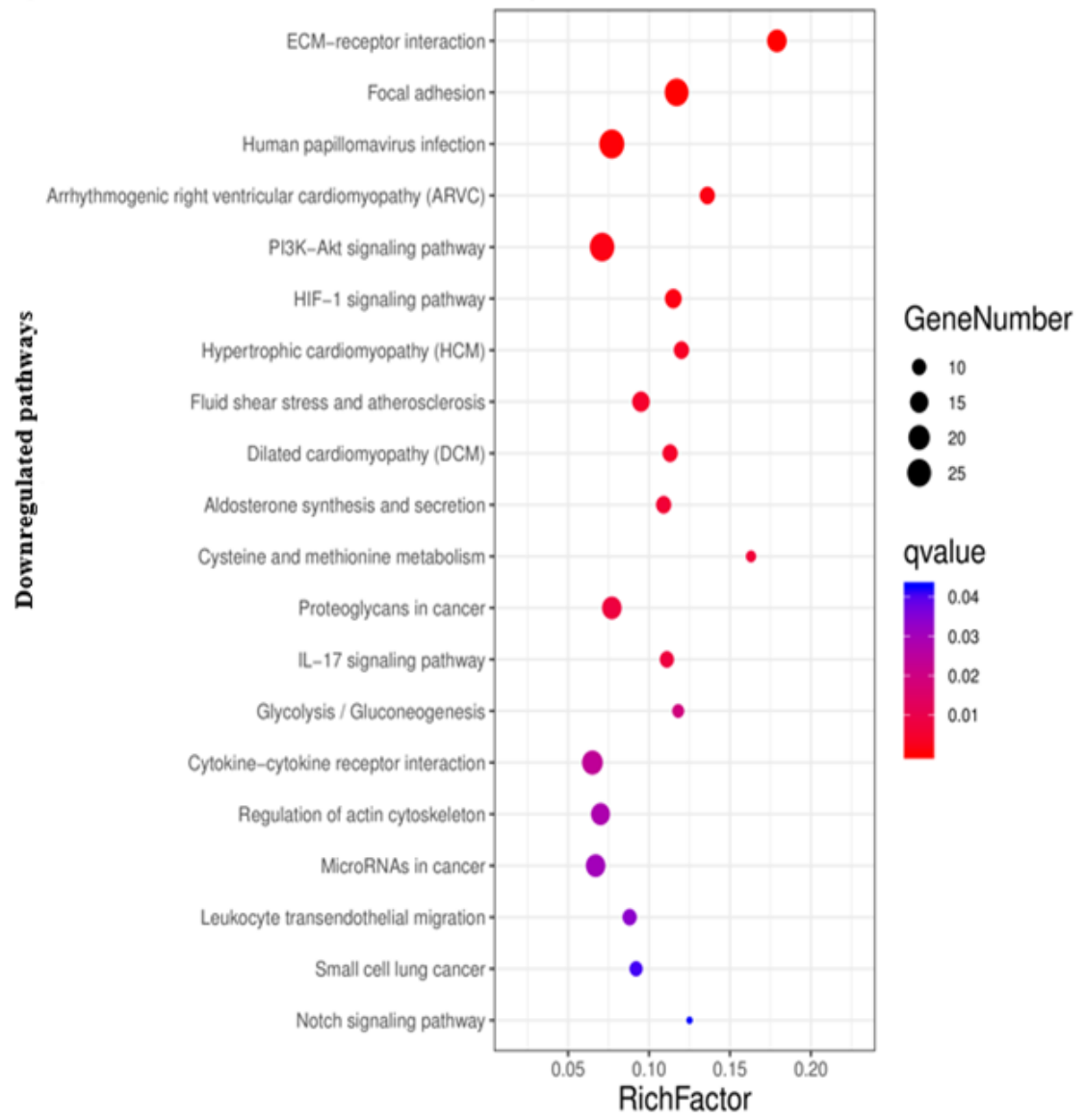

Figure 3 
Expression profile of mRNAs in siDICER1 and NC (SMSCs). (a) Microarray analysis for mRNAs was performed with RNA extracted from siDICER1 $(n=3)$ and NC $(n=3)$ SMSCs. Hierarchical cluster analysis of significantly differentially expressed mRNAs: bright green, under-expression; gray, no change; bright red, over-expression. (b) Ten differentially expressed representative mRNAs were validated in SMSCs siDICER 1 and NC by qPCR ( $\mathrm{n}=10$ per group). GAPDH was used as an internal control. (c) KEGG of the downregulated mRNAs with the top 20 enrichment. Bubble color and size correspond to the $Q$ value and gene number enriched in the pathway. The rich factor indicates the ratio of the number of DEGs mapped to a certain pathway to the total number of genes mapped to this pathway. 
$\mathbf{A}$

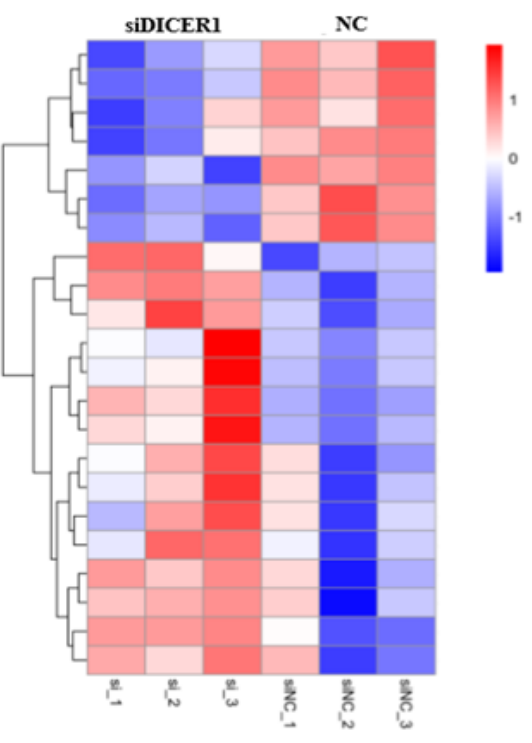

B
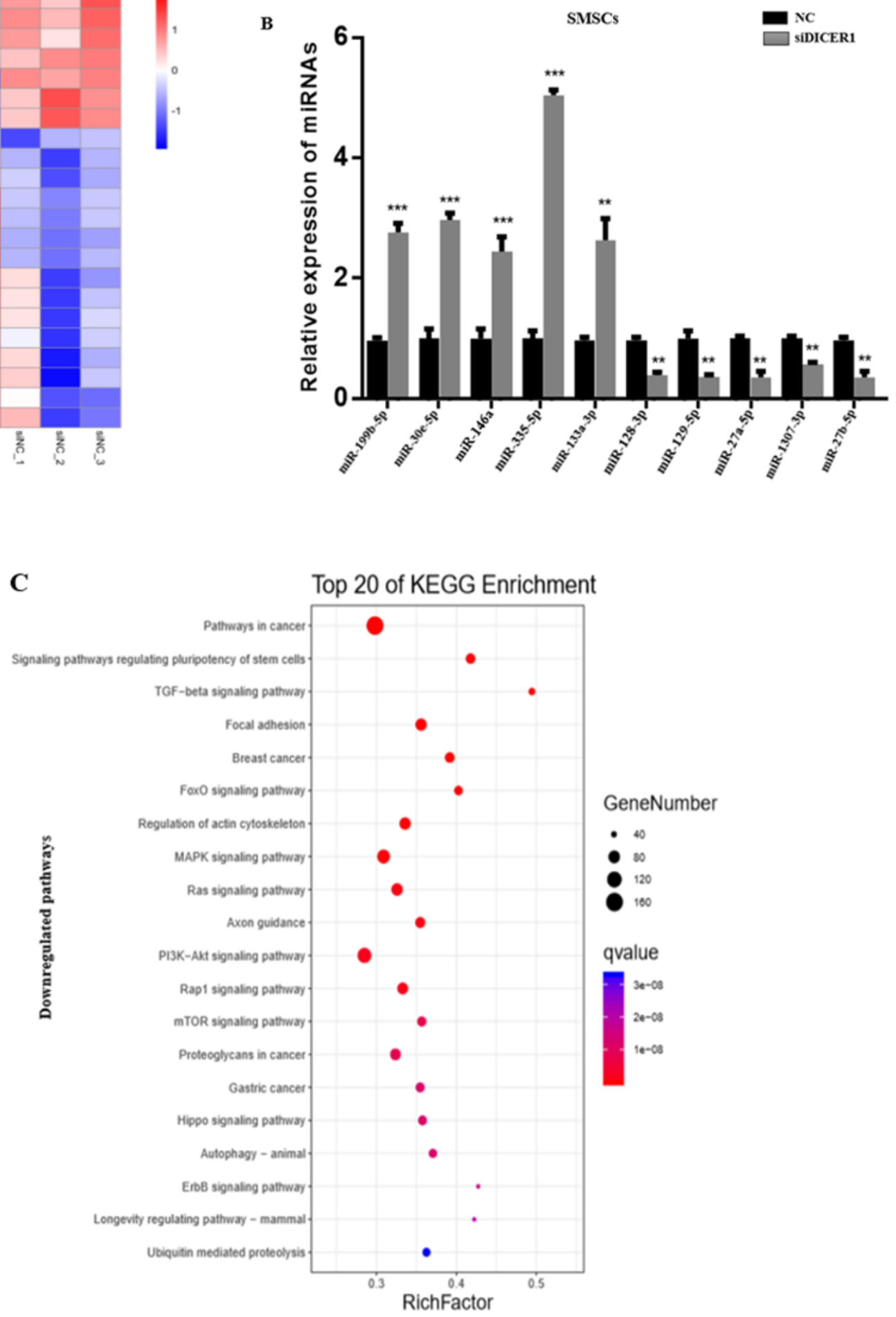

Figure 4

Differentially expressed miRNAs in siDICER1 and NC (SMSCs). (a) Sequencing analysis for miRNAs was performed from siDICER1 $(n=3)$ and NC $(n=3)$ of SMSCs. Hierarchical cluster analysis of significantly differentially expressed miRNAs: bright red, overexpression; white, no change; bright blue, underexpression. (b) Differential expression of ten representative miRNAs was validated in siDICER1 and NC of SMSCs by qPCR ( $\mathrm{n}=10$ per group). (c) KEGG analysis of the upregulated miRNA-mRNA network. Bubble 
color and size correspond to the Q value and gene number enriched in the pathway. The rich factor indicates the ratio of the number of DEGs mapped to a certain pathway to the total number of genes mapped to this pathway.
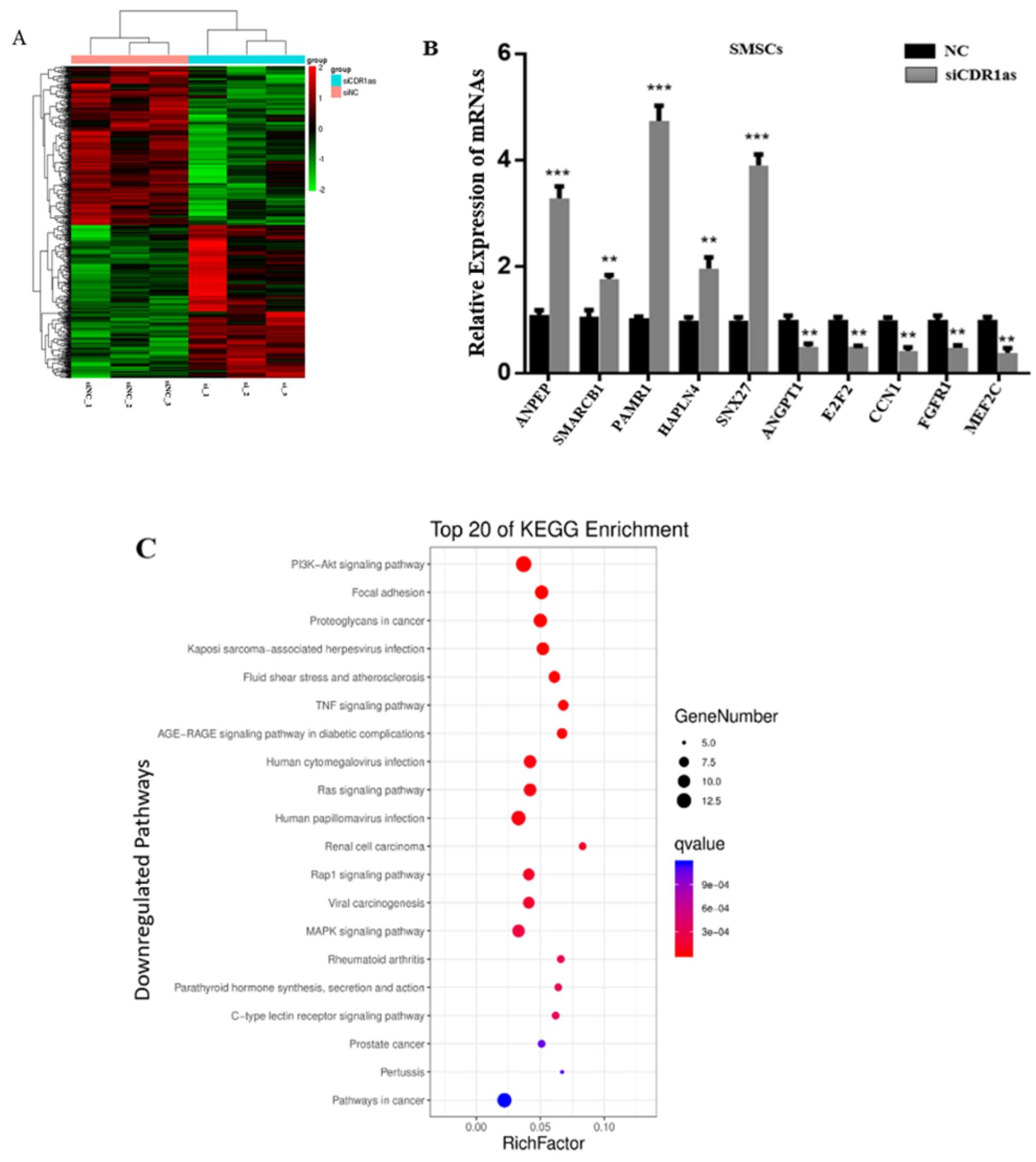

Figure 5

Expression profile of mRNAs in siCDR1as and NC (SMSCs). (a) Microarray analysis for mRNAs was performed with RNA extracted from siCDR1as $(n=3)$ and NC $(n=3)$ SMSCs. Hierarchical cluster analysis 
of significantly differentially expressed mRNAs: bright green, under-expression; gray, no change; bright red, over-expression. (b) Ten differentially expressed representative mRNAs were validated in SMSCs siCDR1as and NC by qPCR ( $n=10$ per group). GAPDH was used as an internal control. (c) KEGG of the downregulated mRNAs with the top 20 enrichment. Bubble color and size correspond to the $Q$ value and gene number enriched in the pathway. The rich factor indicates the ratio of the number of DEGs mapped to a certain pathway to the total number of genes mapped to this pathway.

$\mathbf{A}$

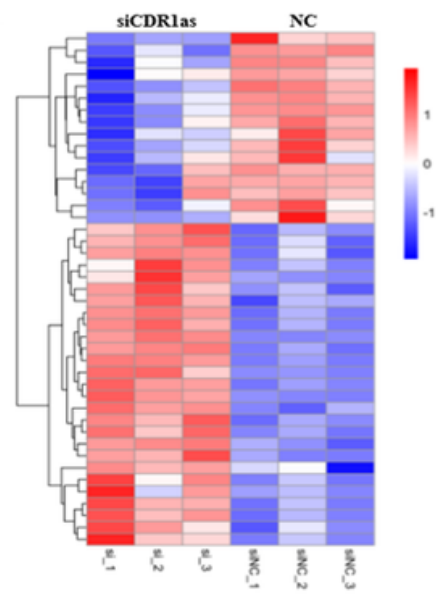

B

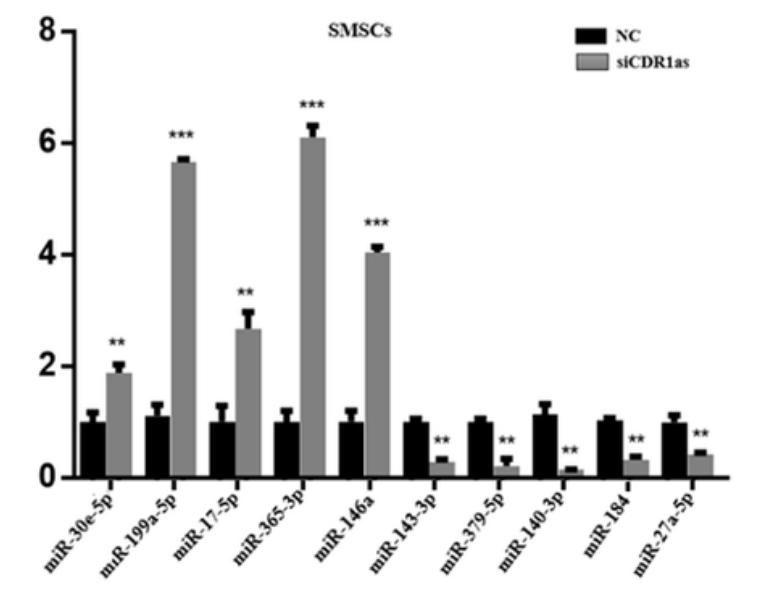

C

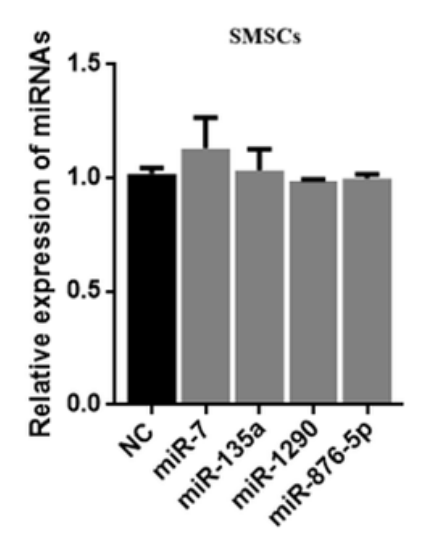

Figure 6

Differentially expressed miRNAs in siCDR1 as and NC (SMSCs). (a) Sequencing analysis for miRNAs was performed from siCDR1as $(n=3)$ and NC $(n=3)$ of SMSCs. Hierarchical cluster analysis of significantly differentially expressed miRNAs: bright red, overexpression; white, no change; bright blue, underexpression. (b) Differential expression of ten representative miRNAs was validated in siCDR1 as and NC of SMSCs by qPCR ( $n=10$ per group). (c) Validation of CDR1as-sponged miRNAs in SMSCs using qPCR analysis after the transfection of siCDR1as. 

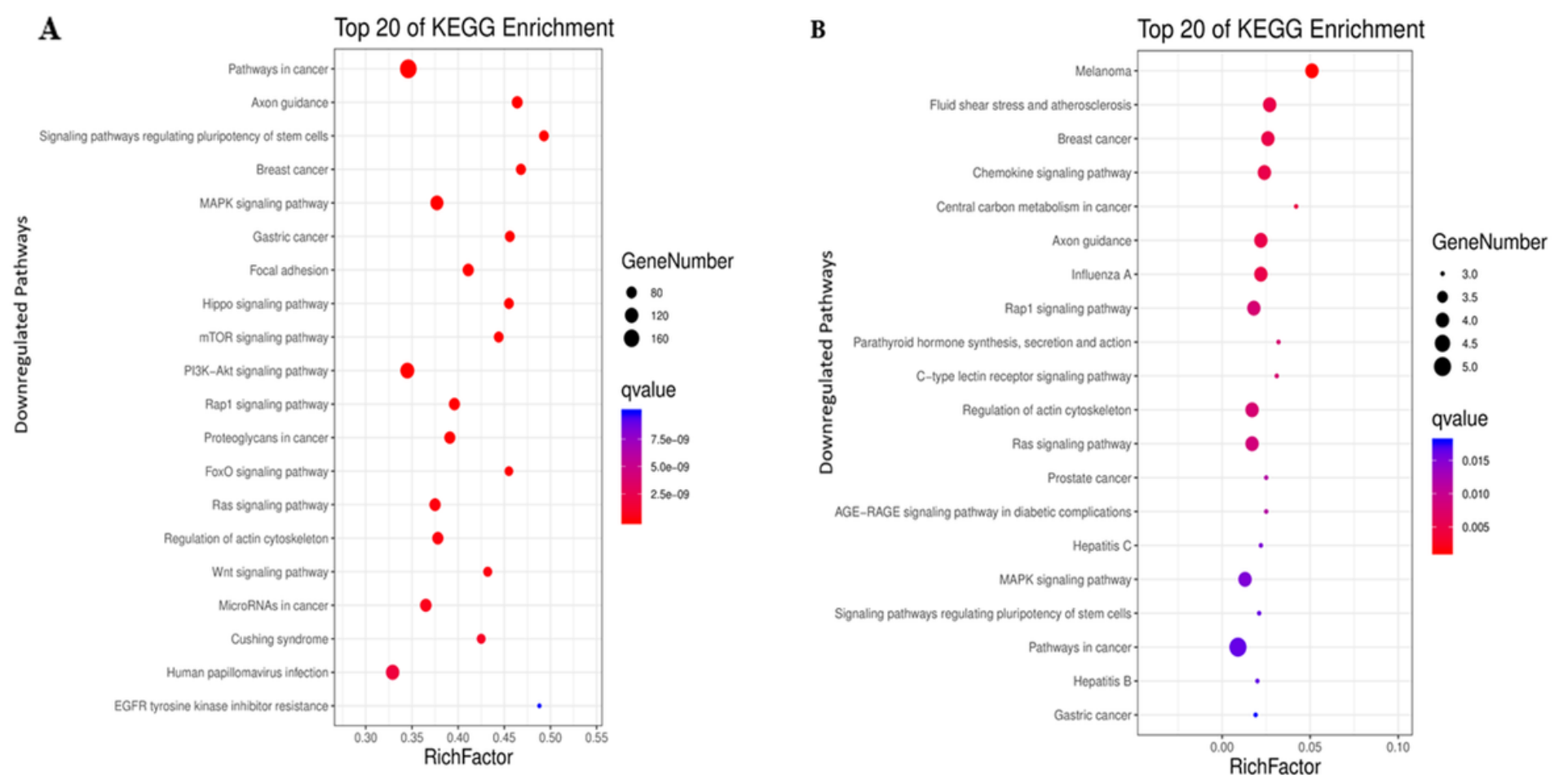

\section{Figure 7}

KEGG analysis of the miRNA-mRNA network. ( $a$ and $b$ ) KEGG pathway enrichment analysis of downregulated. (a) Upregulated miRNAs targets mRNAs, and downregulated (b) overlapped mRNAs. Bubble color and size correspond to the $Q$ value and gene number enriched in the pathway. The rich factor indicates the ratio of the number of DEGs mapped to a certain pathway to the total number of genes mapped to this pathway. 


\section{A CDR1as-Related miRNAs Predicted Binding Sites on mRNAs}

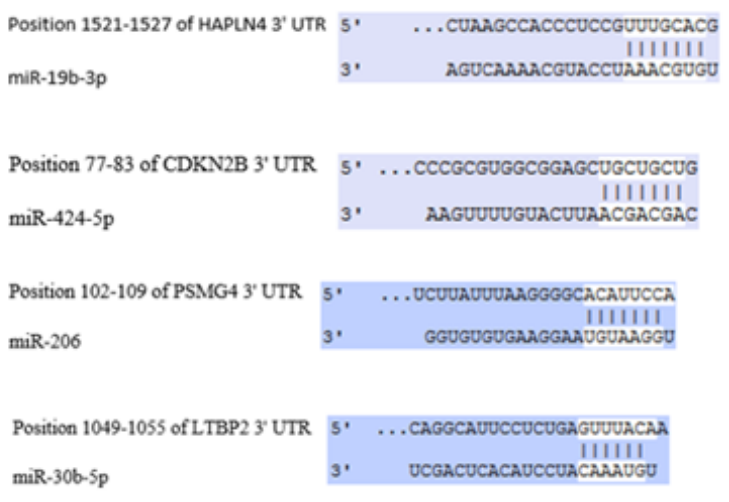

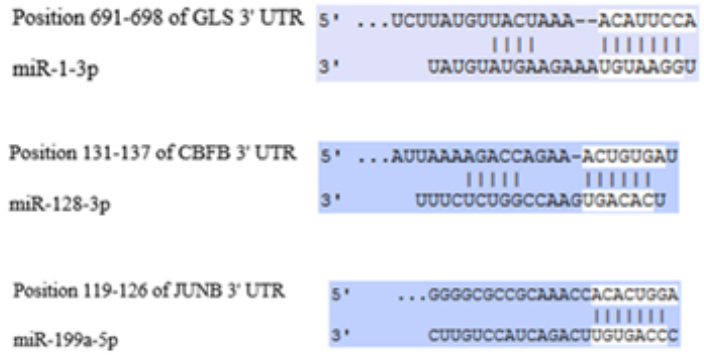

\section{B miRNAs Binding Sites Found on CDR1as}

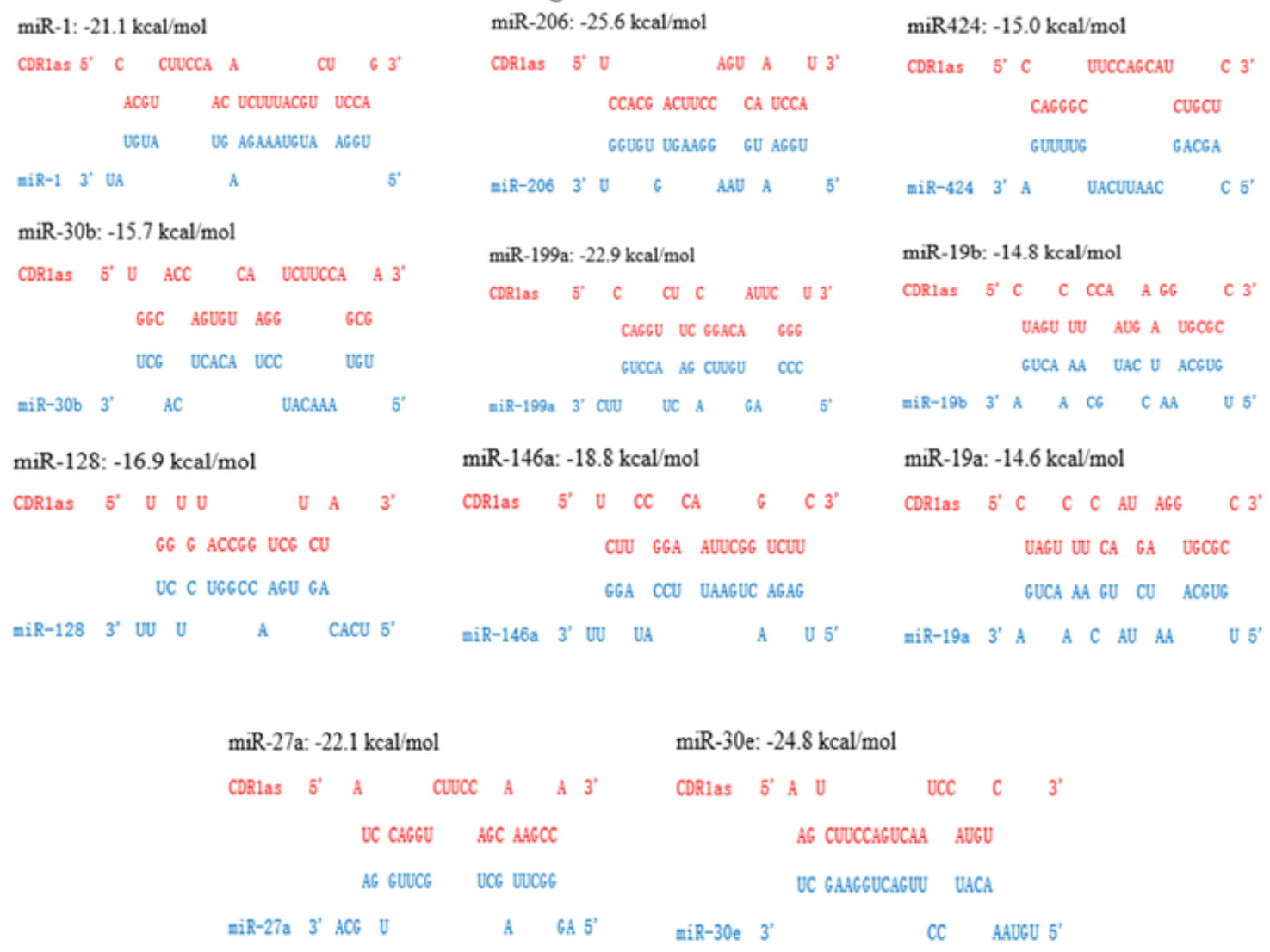

\section{Figure 8}

Prediction of CDR1as-related miRNAs binding sites. (a) Predicted target sites of CDR1as-related miRNAs on mRNAs using Targetscan. (b) Binding sites of miRNAs found on CDR1as with the use of RNAhybrid.

\section{Supplementary Files}


This is a list of supplementary files associated with this preprint. Click to download.

- Supplementarymaterials.pdf 